\title{
4. Überzeugung
}

Mit diesem Kapitel ist der Hauptteil der Arbeit erreicht. Es bildet den Auftakt für die Fundierung von Wissen durch Können im Sinne von „blindem Regelfolgen“. Zu diesem Zweck werde ich zunächst geltend machen, dass Überzeugungen ein irreduzibler Bestandteil von Wissen sind und ihre pragmatische Entsprechung im Äußern und Zuschreiben von Behauptungen finden. Anhand dieser beiden sprachlichen Grundformen werde ich herausarbeiten, dass der Umgang mit Überzeugungen implizit normativ signifikant ist. Das zeigen der Wahrheits- und der Rationalitätsanspruch, die beide mit Behauptungen einhergehen. Im zweiten Teil des Kapitels werde ich ein Erklärungsmodell entwickeln, das diese implizite Normativität des Begriffs der Überzeugung systematisch in den Fokus rückt. Im Rahmen einer normativen Pragmatik werde ich darlegen, dass sich die Praxis des Behauptens und Zuschreibens von Überzeugungen auf einer normativen Beschreibungsebene auf das Einnehmen und Zuweisen von normativen Status zurückführen lässt. Aus der immanenten Perspektive der Diskursteilnehmer bedeutet das, dass beim Umgang mit Überzeugungen anderen gegenüber stets normative Einstellungen eingenommen werden. Die damit einhergehende Unterscheidung in korrekt und inkorrekt manifestiert sich durch die Praxis des Sanktionierens. Auf der Grundlage dieses Erklärungsmodells wird sich anschließend noch detaillierter nachweisen lassen, dass und inwiefern Überzeugungen Können voraussetzen.

\subsection{Wissen und Überzeugung}

Gemäß der Standardanalyse sind Überzeugungen ein wesentlicher definitorischer Bestandteil von Wissen. ${ }^{149}$ Wissen wird üblicherweise als aus Überzeugungen, Rechtfertigungen und Wahrheit zusammengesetzt betrachtet. Statt von Überzeugungen wird gelegentlich auch von Meinungen oder vom Meinen geredet. Anstelle des sperrigen

${ }^{149}$ Vgl. Platon (1994d), 201d - 210a. Platon führt bereits im Menon eine Definition des Begriffs an, ohne sie jedoch zu erörtern. Vgl. Platon (1994a), 98. Der Ausdruck „Standardanalyse“ geht auf M. Williams zurück. Vgl. Williams (2001b), 15 ff. 
„Überzeugungen haben“ wird in der Verbform häufig auch einfach nur „glauben“"gesagt. Das hat im Deutschen zwar auch eine theologische Konnotation. Diese wird jedoch im epistemologischen Zusammenhang zumeist ausreichend deutlich unterdrückt. Ich schließe mich den Konventionen dahingehend an, dass ich all diese Formulierungen als synonym betrachte. Jedenfalls bilden die drei erwähnten Elemente den Kern dessen, was im Allgemeinen unter Wissen verstanden wird. Als Bedingung dafür, dass eine Person weiß, dass $p$, gilt, dass sie glaubt, dass $p$, dass sie darin gerechtfertigt ist zu glauben, dass $p$, und dass es wahr ist, dass $p$. Kurz gesagt ist Wissen gerechtfertigte, wahre Überzeugung.

Ein Schwerpunkt vieler jüngerer Auseinandersetzungen liegt auf der Suche nach weiteren Kriterien. Denn prinzipiell sind Situationen denkbar, in denen diese drei Bedingungen erfüllt sind, ohne dass die Person tatsächlich weiß, dass $p$. Auch ein seltener Zufall kann hierfür verantwortlich sein. ${ }^{150}$ Für eine wirkliche Definition müssen die Bedingungen aber nicht nur zufällig, sondern vielmehr notwendigerweise erfüllt sein. Eine Ergänzung scheint daher angebracht. Um jedoch zu zeigen, dass Wissen durch Können fundiert ist, reicht an dieser Stelle eine Beschränkung auf den dreigliedrigen Kernbestand der Wissensanalyse. Denn was für ihn gilt, bleibt ja auch nach einer Ergänzung durch weitere Bedingungen gültig. Grundsätzlich würde es sogar ausreichen, nur eine der Bedingungen auf Können zurückzuführen, um das Fundierungsverhältnis nachzuweisen. Man könnte sich beispielsweise auf den Nachweis beschränken, dass das Haben von Überzeugungen eine Art Können ist. Allerdings hängt die Überzeugungs- mit der Rechtfertigungsbedingung insofern eng zusammen, als beide auf der gleichen Art von Können basieren. ${ }^{151}$ Was hingegen die Wahrheitsbedingung angeht, so stellt sie sich für die Bestimmung von Wissen nach einer eingehenden Betrachtung letztlich als überflüssig heraus. ${ }^{152}$

Wer vorgibt, etwas $\mathrm{zu}$ wissen, muss zumindest eine entsprechende Überzeugung haben. Wenn jemand beispielsweise weiß, dass Paris die

${ }^{150}$ Gemeint sind die Gegenbeispiele, die auf E. Gettier zurückgehen. Vgl. Gettier (1963). Schon Platon hielt das dreiteilige Ergebnis seiner Analyse für unbefriedigend. Vgl. Platon (1994d), 210a f.

${ }^{151}$ Dies erläutere ich ausführlich in Kap. 5.

${ }^{152}$ Für diese Auffassung argumentiere ich ausführlich in Kap. 6. 
Hauptstadt von Frankreich ist, dann ist davon auszugehen, dass er dies auch glaubt. Und wenn jemand zu wissen vorgibt, dass die Sonne sich um die Erde dreht, so können wir ihm zwar erklären, dass das nicht richtig ist. So lange jedoch, wie ihm dieser Irrtum nicht bewusst ist, hat er diese Überzeugung. Die Bedingung, dass Wissen immer auf Überzeugungen zurückgeht, ist schwer in Zweifel $\mathrm{zu}$ ziehen. Zweierlei könnte man dennoch dagegen einwenden. Einmal könnte man darauf hinweisen, dass wir Dinge sagen wie: „Sie glaubt es nicht, sie weiß es.“ oder „Ich weiß, dass er sie verlassen hat, aber ich kann es nicht glauben." Beides sind völlig korrekte Redensweisen. Allerdings handelt es sich hier um spezielle rhetorische Figuren. Der erste Satz enthält die gleiche Betonung wie: „Sie glaubt es nicht nur, sie weiß es auch.“ Die Überzeugung steht nicht im logischen Gegensatz zum Wissen. Vielmehr wird die größere Gewissheit des Wissens gegenüber der Überzeugung hervorgehoben. Der zweite Beispielsatz bringt eine große Überraschung zum Ausdruck. Dabei wird aber nicht die Überzeugung in Zweifel gezogen, sondern dem Erstaunen, das sie auslöst, Ausdruck verliehen. „Ich kann es nicht glauben.“ ist hier gleichbedeutend mit „Ich kann es nicht fassen.“

Dann könnte man noch ein Gegenbeispiel der folgenden Art formulieren. ${ }^{153}$ Jemand glaubt, nichts über ein bestimmtes Themengebiet zu wissen. Trotzdem gibt er auf Nachfrage eine ganze Reihe richtiger Antworten. Es stellt sich heraus, dass er diese Dinge doch einmal irgendwo gelernt, aber wieder vergessen hat. Ist das eine plausible Erklärung dafür, dass Wissen ohne die Bedingung der Überzeugung auskommt? Der Befragte glaubt, nicht zu wissen, was man ihn fragt. Er weiß also nicht, ob das, was er antwortet, richtig ist. Damit aber ist das, was er tut, nichts anderes als erfolgreiches Raten. ${ }^{154}$ Raten ist aber nicht Wissen. Entweder, jemand rät zufällig, dass etwas der Fall ist, oder er weiß es. Das vermeintliche Gegenbeispiel kann also ebenso wenig überzeugen wie die besagten rhetorischen Figuren.

Man kann vertreten, dass das Meinen oder Haben von Überzeugungen nicht nur für Wissen, sondern für alle Arten mentaler Zustände oder

\footnotetext{
${ }^{153}$ Vgl. Radford (1966).

${ }^{154}$ Vgl. Cohen (1966), 11 u. Lehrer (1968), 496 ff.
} 
Ereignisse eine Grundvoraussetzung ist. ${ }^{155}$ Wenn jemand die Absicht, den Wunsch oder die Befürchtung hat, dass er einmal im Lotto gewinnen wird, dann muss er auch glauben, dass er einmal in Lotto gewinnen wird. Gleiches gilt für Hoffnungen, Erinnerungen, Interessen etc. Das bedeutet nicht, dass all dies auf Überzeugungen zurückgeführt werden kann. Nicht jeder beliebige mentale Zustand ist abhängig von der entsprechenden Überzeugung. Generell ist es aber auch nicht möglich, dass jemand Absichten, Wünsche, Befürchtungen etc. ohne einen gewissen Bestand an Überzeugungen hat. Hinsichtlich des Wissens ist selbst dies noch zu schwach formuliert. Es ist unwahrscheinlich, dass es hier eine Bestimmung geben kann, die völlig ohne Überzeugungen auskommt. ${ }^{156}$ Noch unwahrscheinlicher ist es, dass zusätzlich auf Rechtfertigungen verzichtet werden kann. Ich werde daher im Folgenden von einem Verständnis von Wissen ausgehen, für das die entsprechende Überzeugung eine Minimalbedingung ist.

\section{2 Überzeugungen pragmatisch verstanden}

Für gewöhnlich setzt die Charakterisierung von Überzeugungen bei der allgemeinen Einsicht ein, dass es sich hierbei um mentale Phänomene handelt, die auf einen Gehalt bezogen oder auf einen Gegenstand gerichtet sind. Wir glauben, dass mit der Post ein Brief gekommen ist. Wir glauben an die Gültigkeit der Evolutionstheorie. Oder wir glauben dem Politiker, der uns Steuererleichterungen verspricht. An diesen Beispielen kann man ablesen, dass es immer etwas ist, das wir glauben. Selbst von jemand, der im theologischem Sinne glaubt, kann man noch sagen, dass er an Gott glaubt oder daran, dass es Gott gibt. Aus diesem Grund ist es üblich, Überzeugungen die grundlegende Eigenschaft zuzuschreiben, dass sie intentional sind.

Was aber ist es, worauf sich Überzeugungen beziehen oder richten? Glaubt jemand an die Gültigkeit der Evolutionstheorie, so ist der Gegenstand seiner Überzeugung eben diese Gültigkeit der

${ }^{155}$ Vgl. z. B. Davidson (1982), 321 o. Davidson (1984), 156 f.

${ }^{156} \mathrm{Zu}$ einem Ansatz, wonach Wissen ein grundlegender mentaler Zustand ist, der nicht auf Überzeugung zurückgeführt werden kann, vgl. Williamson (2000), insbes. Kap. 1.4 . 
Evolutionstheorie. Diese Formulierung ist allerdings eher selten und irreführend. Für gewöhnlich glaubt man nicht „einen Gegenstand“, sondern, dass etwas Bestimmtes der Fall ist oder sein wird. Wir glauben nicht „die“ Gültigkeit der Evolutionstheorie oder „den“ Politiker, der uns Steuererleichterungen verspricht. Den Gegenstand unserer Überzeugung drücken wir üblicherweise mit Hilfe eines Dass-Satzes aus. Wir haben die Überzeugung, dass die Evolutionstheorie Gültigkeit hat oder dass der Politiker sein Versprechen hält. Das gilt letztlich auch für elliptisch verkürzte Sätze wie: „Hannah glaubt ihm.“ oder „Karl glaubt, es regnet.“ Wenn wir sie geringfügig umformulieren, erfahren wir auch hier, dass es nicht „er“ ist, was Hannah glaubt, sondern, dass er die Wahrheit sagt. Und Karl glaubt nicht nur „es regnet“, sondern vielmehr, dass es regnet.

Wir verwenden Dass-Sätze, um den Gehalt von Überzeugungen auszudrücken. Dieser ist dabei aber nicht an den Wortlaut eines bestimmten Satzes gebunden. Jemand kann beispielsweise sagen: „Sie glaubt, dass das Mädchen von einem älteren Herrn begleitet wurde." oder „Sie glaubt, dass ein älterer Herr das Mädchen begleitet hat.“ oder auch „She believes that an older gentleman accompanied the girl." Egal ob der Satz im Aktiv oder Passiv oder auf Deutsch oder Englisch formuliert wird, immer drückt er den gleichen Gedanken oder die gleiche Proposition aus. Daher gehen wir davon aus, dass Überzeugungen durch Propositionen individuiert werden. Welche Überzeugung jemand im Einzelnen hat, ist durch den propositionalen Gehalt festgelegt, dem sie entspricht.

Etwas zu glauben, ist aber nur eine Möglichkeit, sich auf einen Gehalt $\mathrm{zu}$ beziehen. Ebenso gut kann man beispielsweise eine Absicht, einen Wunsch oder eine Befürchtung haben. Letztlich lässt sich auch Wissen nach diesem Schema charakterisieren. Zumindest legt dies die Rede von propositionalem Wissen sowie dessen Rückbindung an Überzeugungen nahe. So kann ich einerseits glauben, dass ich einmal im Lotto gewinnen werde. Andererseits kann ich aber auch die Absicht oder den Wunsch haben, einmal im Lotto $\mathrm{zu}$ gewinnen. Unter den entsprechenden Voraussetzungen ist es sogar möglich, dass ich befürchte oder weiß, dass ich einmal im Lotto gewinnen werde. In all diesen Fällen ist es derselbe propositionale Gehalt, zu dem ich aber immer auf eine andere Art und Weise in Relation stehe. Daher lassen sich diese unterschiedlichen Formen 
der Bezugnahme auf einen Gegenstand unter dem Terminus der propositionalen Einstellung zusammenfassen.

Im Großen und Ganzen ist diese Charakterisierung von Überzeugungen vertraut und unkontrovers. Dennoch werde ich ihr eine alternative Sichtweise entgegensetzen. Der Grund dafür ist, dass diese Darstellung bereits von Anfang an intellektualistisch und mentalistisch angelegt ist. Sie ist vor allem an dem orientiert, worauf mentale Phänomene wie Überzeugungen, Absichten oder Wissen bezogen oder gerichtet sind. Als charakteristisch für Überzeugungen gilt der Bezug zu einem propositionalen Gehalt. Damit aber ist bereits eine ganz bestimmte Untersuchungsrichtung vorgegeben. Was also beispielsweise die Erklärung von Können betrifft, so wird sie aller Voraussicht nach auf der Grundlage von propositionalem Wissen beziehungsweise Gehalt aufbauen. Daraus aber ergibt sich dann genau das Erklärungsmodell, das sich zuvor als regressiv herausgestellt hat.

Die Sichtweise, die ich dem gegenüberstellen werde, ist grundlegend anders ausgerichtet. Sie setzt bei einer genauen Analyse des Sprachgebrauchs an und ist damit erst einmal an dem orientiert, was wir tun, wenn wir Überzeugungen haben. Primär gelten Überzeugungen also nicht als propositionale Einstellungen, sondern als etwas, das sich in Sprechhandlungen konstituiert. Diese Grundeinsicht ermöglicht es in einem ersten Schritt, die implizite Normativität aufzuzeigen, die den Umgang mit Überzeugungen prägt und ausmacht. Ein erster zentraler Gedanke für die zu entwickelnde normative Pragmatik ist daher der, dass die sprachliche Praxis insofern implizit normativ ist, als sie eine grundlegende normative Signifikanz aufweist. Diesen werde ich im Folgenden dadurch vorbereiten, dass ich den Umgang mit Überzeugungen in zwei Momente aufgliedern und analysieren werde. Als die sprachlichen Grundformen des Meinens oder Habens von Überzeugungen werde ich das Behaupten oder Äußern von Behauptungen auf der einen Seite und das Zuschreiben von Behauptungen auf der anderen ausweisen. In einem zweiten Schritt wird es dann darum gehen, dass Überzeugungen eine Bedeutung haben. Im Rahmen einer inferentiellen Semantik wird es möglich zu erklären, wann und wodurch sprachliche Interaktion bedeutsam wird. Pragmatik und Semantik sind somit die explanatorische Grundlage für Gehalt und Wissen und nicht etwa umgekehrt. Wenn im Folgenden 
dennoch von propositionalem Gehalt die Rede ist, dann nur deshalb, weil die Erklärung der systematischen Entwicklung der Untersuchung gelegentlich vorausgreift.

\subsection{Behaupten}

Der beste Hinweis auf eine Überzeugung ist der, dass eine Person eine solche offen bekundet. Eine Überzeugung liegt offensichtlich dann vor, wenn sie im Medium der Sprache als Behauptung veröffentlicht wird. Im Sprechakt der Behauptung finden Überzeugungen ihre pragmatische Entsprechung. Was immer sprachliche Äußerungen noch sein mögen, in ihrer elementarsten Form sind sie Behauptungen, die eine Überzeugung zum Ausdruck bringen. Die These ist somit die, dass Satzäußerungen, in denen Überzeugungen in Form von Behauptungen kundgetan werden, das kleinste Element der sprachlichen Praxis sind. Mit Behauptungen beginnt jeglicher sinnvolle Sprachgebrauch.

Man könnte einwenden, dass es auch Überzeugungen gibt, die nicht öffentlich kundgetan werden. Manchmal ist es sogar besser, eine Überzeugung für sich zu behalten, etwa wenn jemand glaubt, dass ein Mitspieler beim Pokern blufft oder dass die Uhr des Flohmarktverkäufers eigentlich das Doppelte wert ist. Über Meinungen in Form von privaten Erlebnissen oder inneren Monologen zu spekulieren, ist allerdings nur dann sinnvoll, wenn eine Sprache vorausgesetzt wird, in der diese auch mitgeteilt werden können. Sprache muss dabei in erster Linie als ein öffentliches und soziales Phänomen begriffen werden. Eine rein private Sprache kann es nicht geben, weil sie für niemanden verständlich wäre. ${ }^{157}$ Daher kann es private Überzeugungen nur vor dem Hintergrund sozialer Kommunikationspraktiken geben. Grundsätzlich kann etwas also nur dann den Status einer Überzeugung haben, wenn es auch Teil des öffentlichen Diskurses sein kann.

Man könnte aber auch darauf hinweisen, dass es neben Behauptungen noch andere Arten gibt, Überzeugungen zu äußern. Müssen wir nicht auch Fragen, Befehle, Lügen, Metaphern, Ironie oder die indirekte Rede berücksichtigen? Mit Sicherheit handelt es sich hierbei um wichtige und

${ }^{157}$ Vgl. Wittgenstein (1984d), §§ 202, 244-271 u. 293. 
interessante Sprachformen. Allerdings spielen sie im Vergleich zu Behauptungen eine untergeordnete und abgeleitete Rolle. Denn ohne die aufrichtige und direkte behauptende Rede sind auch sie nicht möglich. Die kommunikative Grundsituation liegt weder in der vereinzelten Sprechhandlung noch im Formulieren von Fragen, Befehlen, Lügen oder Metaphern. Vielmehr besteht sie aus den komplementären Reaktionen von Diskursteilnehmern auf sprachliche Äußerungen mit dem Status von Behauptungen. Nur weil ein Satz behauptend gebraucht werden kann, kann er wirklich verstanden und in Form einer Frage oder Metapher verwendet oder als Lüge oder ironische Bemerkung reformuliert werden. Denn es sind Behauptungen und nicht etwa Fragen, Befehle, Lügen oder Metaphern, die in Folgerungsbeziehungen stehen und somit Bedeutung generieren. ${ }^{158}$

Schließlich könnte man noch einwenden, dass es auch subsententiale Einheiten wie Wörter, Ausdrücke, Namen oder Begriffe gibt. Ist dies nicht die basalste Ebene des Sprachgebrauchs? Eine angemessene Argumentation für den Gedanken, dass es vornehmlich Sätze sind, mit denen wir Sprechhandlungen vollziehen, würde $\mathrm{zu}$ weit von dem eigentlichen Anliegen dieser Arbeit wegführen. Daher muss der Verweis auf einige prominente Positionen aus der Literatur genügen, um ihn zu stützen. ${ }^{159}$ Der Gedanke lässt sich letztlich bis $\mathrm{zu}$ Immanuel Kant zurückverfolgen. Auf ihn geht die Einsicht zurück, dass die kleinste Einheit, die der Verstand im Denk-, Verstehens- oder Erkenntnisakt begreifen kann, das Urteil ist. ${ }^{160}$ Urteile sind grundlegender als Begriffe, da Begreifen letztlich immer Urteilen ist. Der Verstand bedient sich eines Begriffs, indem er durch ihn urteilt.

Diese Idee greifen Frege und Wittgenstein auf und analysieren sie in sprachlich-logischem Zusammenhang. Eine bedeutende Einsicht Freges ist die, dass Wörter nur im Zusammenhang eines vollständigen Satzes eine Bedeutung haben. ${ }^{161}$ Das gilt selbst dann, wenn wir vermittels einzelner Worte kommunizieren. Ausrufe wie „Aua!“, Befehle wie „Platte!“ oder

${ }^{158}$ Vgl. hierzu auch Kap. 5.

${ }^{159}$ Weitere, semantische Gründe für ein Vorrang des Propositionalen gebe ich in Kap. 5.3 u. 5.4.

${ }^{160}$ Vgl. Kant (1998), B93 (A68) f. Vgl. auch Brandom (1994), 79 ff.

${ }^{161} \mathrm{Vgl}$. Frege (1988), §§ 46, 60 u. 62. 
auch frühkindliche Einwortsätze wie „Teddy.“ stehen dieser Ansicht nicht entgegen. Denn auch bei ihnen handelt es sich um Sätze. ${ }^{162}$ Allerdings sind sie „,degeneriert“ oder elliptisch verkürzt. In einem sinnvollen sprachlichen Zusammenhang bedeutet „Aua!“ soviel wie „Ich habe Schmerzen.“, „Platte!“ soviel wie „Bring mir eine Platte!“ und „Teddy.“ soviel wie „Da ist mein Teddy." Der Sprachgebrauch entscheidet darüber, wie genau die einzelnen Satzfragmente ergänzt werden müssen und was bestimmte Einwortsätze bedeuten. Ein Satz mag sich aus einzelnen Wörtern zusammensetzen, die sich isoliert betrachten und darstellen lassen. Es ist aber allein der Satz, dessen Äußerung einen Zug im Sprachspiel darstellt. Kurz: Der sinnvolle Gebrauch von Sprache beginnt mit ganzen Sätzen. In ihrer elementarsten Form bringen sie Überzeugungen zum Ausdruck und so lässt sich vorausgreifend hinzufügen - fungieren dadurch als Gründe für andere Überzeugungen.

\section{4 Überzeugungen zuschreiben}

Dass Überzeugungen in offenen Behauptungen Ausdruck finden, ist wesentlich weniger spekulativ, als sie beispielsweise in inneren Monologen $\mathrm{zu}$ vermuten. Die sprachliche Praxis ist weitestgehend unverstellt. Dies gilt gleichermaßen für Überzeugungen, die nicht durch Sprecher selbst, sondern durch die Zuschreibung anderer Gesprächsteilnehmer in den Blick geraten. Wenn wir die kommunikative Situation als Ganze berücksichtigen, erkennen wir auf der einen Seite Sprecher, die selbst etwas behaupten. Auf der anderen Seite gibt es aber auch Zuhörer oder Interpreten, die feststellen, dass andere etwas glauben. Dabei handelt es sich nicht notwendigerweise um zwei völlig verschiedene Sachverhalte. Ein Sprecher ist prinzipiell immer auch Zuhörer und Interpret anderer. Dadurch ist gewährleistet, dass sich verschiedene Sprecher zumindest gelegentlich auf dieselben Überzeugungen beziehen.

Mit der Berücksichtigung von Zuschreibungen von Überzeugungen bekommt die Betrachtung eine soziale Dimension. Damit wird der Tatsache Rechnung getragen, dass Sprache und Kommunikation ebenfalls eine grundsätzlich soziale Angelegenheit ist. Diskursive Praktiken sind im

${ }^{162}$ Vgl. Wittgenstein (1984d), §§ 19-22 u. 49 
Wesentlichen interpersonale Praktiken. Die Elemente einer diskursiven Gemeinschaft sind die einzelnen, miteinander kommunizierenden Sprecher. Die diskursive Praxis weist somit immer eine Ich-Du-Struktur auf, aus der sich eine (erweiterte) diskursive Gemeinschaft, vorgestellt als eine Ich-Wir-Beziehung, erst ableitet. Für eine überzeugende Rekonstruktion der Normativität im Umgang mit Überzeugungen ist es entscheidend, auf der Ebene von Ich-Du-Beziehungen zwischen einzelnen Sprechern zu verbleiben. ${ }^{163}$

Der Ich-Aspekt der sprachlichen Übermittlung von Überzeugungen ist recht unproblematisch und leuchtet intuitiv ein. Ein Sprecher, der Überzeugungen hat wie beispielsweise „Mit der Post ist ein Brief gekommen.“ oder „Die Evolutionstheorie ist gültig.“, kann diese in Form von gleichlautenden Behauptungen äußern. Die Überzeugungen

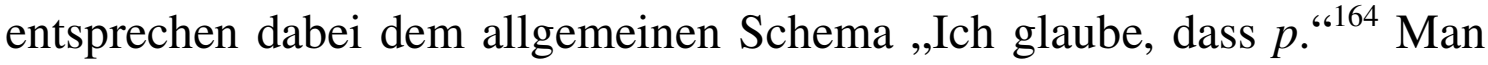
kann dies auch als die Perspektive der ersten Person bezeichnen. Auch der Du-Aspekt ist nicht wesentlich komplizierter. Sätze wie „Du behauptest, dass mit der Post ein Brief gekommen ist.“ oder „Du glaubst, dass die Evolutionstheorie gültig ist." sind unkritische Beispiele dafür, wie Überzeugungen auch durch Zuschreibung zum Ausdruck kommen können. Das allgemeine Schema, dem sie entsprechen, lautet hier: „Du glaubst, dass $p$. " Dies kann man als die Perspektive der zweiten Person bezeichnen.

Man könnte sich nun allerdings fragen, warum die Berücksichtigung dieser zweiten Perspektive bei der Erklärung von Überzeugungen relevant sein soll. Die Beschäftigung mit einfachen Behauptungen scheint doch vollkommen ausreichend. Jedenfalls ist die Annäherung an mentale

${ }^{163} \mathrm{Zu}$ der Auffassung, dass die grundlegende Struktur einer diskursiven Gemeinschaft eine Ich-Du- und nicht eine Ich-Wir-Beziehung ist, vgl. auch Brandom (1994), 38 f., 508 u. $598 \mathrm{ff}$. R. Brandom bezeichnet erstere als symmetrisch und anti-autoritativ. Die Annahme einer privilegierten Vogelperspektive hält er weder für hilfreich noch für zutreffend. Dies entspricht auch D. Davidsons Verständnis von sprachlicher Praxis als wechselseitiger Interpretation. Vgl. z. B. Davidson (1984), Kap. 9 u. 11. Zu einer Diskussion von Einwänden gegen eine soziale Theorie der Bedeutung in Begriffen einer Ich-Wir-Beziehung vgl. Esfeld (2002), $121 \mathrm{ff.} \mathrm{u.} 164 \mathrm{ff}$.

${ }^{164}$ Häufig entsprechen Äußerungen der Art „Ich glaube, dass $p$.“ denen der Art , $p$ “. Um jedoch auszuschließen, dass es sich um Wünsche, Befürchtungen, Erinnerungen, Lügen etc. handelt, ist die ausführliche Formulierung ratsam. 
Phänomene auf der Grundlage von Zuschreibungen eher ungewöhnlich. Zunächst sei daher erneut darauf hingewiesen, dass eine Erklärung, die auf die sprachliche Praxis rekurriert, auf eine soziale Perspektive angewiesen ist. Sprache als rein privates Phänomen ist nicht denkbar. Mit Behauptungen allein lässt sich aber keine wirklich soziale Situation rekonstruieren. Das Moment der Zuschreibung garantiert hier die unmittelbare Bezugnahme auf andere Diskursteilnehmer. Analog verhält es sich mit Normativität, um die es ja ebenfalls gehen soll.

Um einen Eindruck von der Relevanz und dem Potential der Perspektive der zweiten Person zu bekommen, sind zudem Daniel Dennetts Überlegungen zu Intentionalität äußerst aufschlussreich. ${ }^{165}$ Dennetts Idee ist nämlich die, Intentionalität generell in Begriffen der Zuschreibung zu verstehen. Wenn also Überzeugungen paradigmatische Fälle von intentionalen Zuständen sind, dann muss das für sie in besonderem Maße gelten. Da es mir in erster Linie genau um diese geht, werde ich im Folgenden Phänomene wie Wünsche, Hoffnungen, Befürchtungen etc. nicht berücksichtigen. Dabei kann auch die Frage nach dem Gehalt intentionaler Zustände zunächst einmal außen vor bleiben. Was zählt, ist der Gedanke, dass Intentionalität eine von außen zugeschriebene Eigenschaft ist, die vom Standpunkt desjenigen festestellt werden kann, der eine bestimmte Vorhersagestrategie anwendet.

Dennett zufolge heißt, jemandem oder etwas Überzeugungen zuzuschreiben, ihm gegenüber eine, ,intentionale Einstellung“ einzunehmen. ${ }^{166}$ Eine solche Einstellung einzunehmen heißt, den Gegenstand der Betrachtung in der Praxis als ein ,intentionales System“ zu behandelt. Dabei ist es vom Standpunkt des zuschreibenden Interpreten belanglos, ob es sich um eine Person, eine Pflanze, ein Tier oder auch um ein Artefakt wie beispielsweise einen Schachcomputer handelt. Sobald das Verhalten eines Systems Rückschlüsse auf Intentionalität zulässt, lässt es sich als ein intentionales etablieren. Und dies ist genau dann der Fall, wenn die Zuschreibung von intentionalen Zuständen dabei hilft, das Verhalten des jeweiligen Systems zu erklären und vorauszusagen. In der Tat ist dies eine Strategie, die wir im täglichen Sprachgebrauch immer wieder verfolgen.

\footnotetext{
${ }^{165}$ Vgl. zum Folgenden insbes. Dennett (1971) u. Dennett (1987), Kap. 2.

${ }^{166}$ Vgl. Dennett (1971), 90.
} 
Nicht selten sagen wir Dinge wie: „Pflanzen wollen immer zum Licht.“, „Der Hund glaubt, dass die Katze auf den Baum geflüchtet ist.“ oder „Der Computer denkt, dass du ins Internet willst, wenn du den Browser öffnest.“ Oft ist dies nicht nur eine nützliche, sondern zugleich die einzige Erklärung, die wir geben können. Daher sollten wir diese Perspektive bei der Analyse von Überzeugungen nicht unterschätzen. $\mathrm{Ob}$ es sinnvoll und gerechtfertigt ist, Tiere, Pflanzen oder Artefakte als vollwertige Teilnehmer am Sprachspiel der Überzeugung anzusehen, ist damit nicht entschieden.

Das Interessante an Dennetts Ansatz ist, dass Spekulationen über innere Zustände, Substanzen oder ähnliches bewusst ausbleiben. Die intentionale Einstellung ist eine zugleich pragmatische und antiintellektualistische Erklärungsstrategie, die jeden Mentalismus oder Repräsentationalismus vermeidet. ${ }^{167}$ Bevor darüber zu entscheiden ist, ob eine Person über Merkmale wie einen Geist, eine Seele, Selbsterkenntnis oder mentale Repräsentationen verfügt oder ob es einen Dualismus aus physikalischen und mentalen Substanzen gibt, steht mit dieser Strategie bereits ein aussagekräftiges Mittel für die Erklärung mentaler Zustände wie Überzeugungen zur Verfügung. Man tut nichts weiter, als von dem Auftreten intentional erklärbaren Verhaltens auf das Vorliegen mentaler Zustände zu schließe. Das entzieht nicht nur dem cartesianischen Skeptizismus, sondern auch jeglicher Variante eines intellektualistischen Regresses die Grundlage. Zugleich kann diese Strategie durchaus mit materialistischen Intentionalitätstheorien, wie sie von den Neurowissenschaften oder der Hirnforschung vorgeschlagen werden, in Einklang gebracht werden.

Dennetts These ist eine stärkere, als ich sie hier vertreten werde. Er behauptet, dass sich alles und jeder ausschließlich aus der Perspektive der Zuschreibung als intentionales System ausweisen lässt. Demnach gibt es für ihn Überzeugungen nur aus der Perspektive der zweiten Person. Ich vertrete dagegen die schwächere These, wonach Überzeugungen einem Sprecher zugeschrieben oder von ihm in Behauptungen geäußert werden. Aus Dennetts Sicht muss dies wie ein Rückschritt wirken. Mit Blick auf

${ }^{167}$ Vgl. auch Rorty (1979), Kap. 1.5 o. Esfeld (2002), Kap. 4.1. D. Dennett deutet die Vorteile seiner Theorie dieser Tradition gegenüber nur an. Vgl. Dennett (1971), $100 \mathrm{f}$. 
die sprachliche Praxis dagegen ist seine Sicht $\mathrm{zu}$ restriktiv und daher unvollständig. Denn zum einen ist die Perspektive der Zuschreibung allein ebenso wenig eine soziale, wie es die der Behauptung allein ist. Zum anderen sind Zuschreibungen von Überzeugungen nicht weniger problematisch oder unproblematisch als offene Behauptungen. Insofern also die Perspektive der ersten Person bei Dennett nicht vorkommt, bleibt sein Vorschlag letztlich hinter meinem sprachpragmatischen Ansatz zurück. Die Perspektive der zweiten Person ist eine sinnvolle und notwendige Ergänzung der Perspektive der ersten Person, nicht aber ihr Ersatz.

\subsection{Die normative Signifikanz von Überzeugungen}

Mit der Darstellung der wichtigsten und elementarsten deskriptiven Merkmale des Sprachspiels der Überzeugung wird es nun möglich, die diskursive Praxis auf ihre fundamentale normative Dimension hin $\mathrm{zu}$ interpretieren. Ich werde darlegen, dass sich Sprecher beim Behaupten und Zuschreiben von Überzeugungen immer auch beurteilen dahingehend, ob ihr Verhalten den Erwartungen entspricht oder nicht. Der Umstand, dass eine Behauptung als Überzeugung ein offenes Fürwahrhalten ist, macht sie zu etwas grundsätzlich Bewertbarem. Es lässt sich beurteilen, ob jemand dem Wahrheitsanspruch, den er mit seiner Behauptung erhebt, auch gerecht wird. Zudem können Zuschreibungen von Überzeugungen als eine Form der Bewertung interpretiert werden. Mit Hilfe von Dennetts Strategie der ,intentionalen Einstellung“ lässt sich zeigen, dass dabei auf substantielle Weise Rationalität vorausgesetzt wird. Denn indem wir jemandem Überzeugungen zuschreiben, beurteilen wir ihn immer auch dahingehend, ob er sich rational verhält oder nicht. So stellt sich sowohl die Praxis des Behauptens als auch die des Zuschreibens von Überzeugungen als grundlegend und auf bedeutsame Weise normativ aufgeladen heraus.

\subsection{Wahrheit normativ verstanden}

Ein wichtiger Grund für die normative Aufgeladenheit des Behauptens und Zuschreibens von Überzeugungen liegt in dem unmittelbaren 
Zusammenhang von Überzeugung und Wahrheit. Das lässt sich schon allein daran erkennen, dass wir das Meinen oder Haben von Überzeugungen auch als Fürwahrhalten bezeichnen. ${ }^{168}$ Als beispielsweise Kopernikus behauptete, dass sich die Erde um die Sonne dreht, hielt er dies (natürlich) für wahr. Viele seiner Zeitgenossen waren anderer Meinung. Sie hielten für wahr, dass sich die Sonne um die Erde dreht. Nun besteht die diskursive Praxis aber nur in den seltensten Fällen aus einzelnen, isolierten Behauptungen. Vielmehr reagieren Diskursteilnehmer auf Behauptungen anderer mit komplementären Sprechhandlungen. Wer eine Überzeugung äußert, kann damit rechnen, dass sich jemand anderes mit einer anderen Überzeugung darauf bezieht. Das kann eine inhaltliche Ergänzung oder die Weiterführung eines Gedanken sein. Es kann aber auch sein, dass für die Behauptung eine Begründung eingefordert wird.

Grundsätzlich ist es also nicht gleichgültig, wie jemand zu dem steht, was er für wahr hält. Vielmehr ist das Meinen oder Haben einer Überzeugung gleichbedeutend damit, eine bestimmte Einstellung oder Haltung einzunehmen. Daher auch die Konvention, es als eine propositionale Einstellung zu bezeichnen. Jemand, der etwas glaubt, nimmt eine affirmative Haltung ein in Bezug auf das, was er glaubt. Nichts anderes besagt die Rede vom Fürwahrhalten. Wenn jemand eine Überzeugung äußert, dann verbindet sich damit der Anspruch, dass dies wahr ist. Wer etwas behauptet, erhebt implizit immer einen Wahrheitsanspruch. ${ }^{169}$ Das heißt nicht, dass das Meinen ein freier Entschluss ist. Eine Überzeugung zu haben, ist nicht der Vorgang oder das Resultat einer (willkürlichen) Entscheidung. Wir haben nicht eine Überzeugung, und dann entscheiden wir noch darüber, ob wir sie für wahr oder falsch halten. ${ }^{170}$ Dann müsste es ja auch möglich sein, der unliebsamen Neigung zu falschen Überzeugungen zu widerstehen. Ebenso wenig heißt es, dass das, was jemand glaubt, automatisch wahr ist. Niemand ist in der Lage, ausschließlich das zu glauben, was auch

\footnotetext{
${ }^{168}$ Vgl. z. B. Kant (1998), B848 ff. (A820 ff.) o. Frege (1993), 30 ff.

$169 \mathrm{Vgl}$. Tugendhat/Wolf (1983), 24 u. 221. Das ist auch die Grundlage für das Äquivalenzschema, das im Zentrum der deflationistischen Wahrheitstheorie steht. Vgl. Kap. 6.8 insbes. Anm. 324.

${ }^{170}$ Vgl. Wittgenstein (1984d), 514.
} 
tatsächlich wahr ist. ${ }^{171}$ Aber indem jemand eine Überzeugung hat, zieht er etwas in Betracht mit dem Anspruch, dass es wahr ist, oder etwas anders gesagt, was aus seiner Sicht wahr sein soll.

Der Zusammenhang von Wahrheit und Überzeugung findet sich aber nicht nur in der Perspektive der ersten Person. Er spiegelt sich in Form einer Erwartungshaltung in der Perspektive der zweiten Person. Stellen wir uns die kommunikative Standardsituation vor, in der jemand eine Überzeugung äußert. Anders als bei Kopernikus' These vom Heliozentrismus gehen wir meistens, wenn auch nicht immer, davon aus, dass die Behauptungen anderer wahr sind. Auch wir halten in den meisten Fällen für wahr, was sie behaupten. Gelegentlich ist Zweifel zwar durchaus angebracht und wünschenswert. Irrtümer sind immer möglich. Die überwiegende Mehrheit der Behauptungen anderer für falsch zu halten, würde jedoch zu keiner sinnvollen Gesprächssituation führen.

Donald Davidson hat diesen Umstand zum Prinzip erklärt. Das durch ihn prominent gewordene „Nachsichtigkeitsprinzip“ (Principle of Charity) besagt, dass die Überzeugungen eines jeden Sprachbenutzers, um überhaupt verständlich sein zu können, zum größten Teil wahr und konsistent sein müssen. ${ }^{172}$ Dass sich ein Sprecher irrt, kann zwar keinesfalls ausgeschlossen werden. Unterstellen wir ihm aber $\mathrm{zu}$ viele Irrtümer, so untergraben wir unsere Fähigkeit zu verstehen, worin diese eigentlich bestehen. Davidson formuliert mit dem Gedankenexperiment der „radikalen Interpretation“ eine paradigmatische Sprecher-Hörer-Situation, worin ein Interpret versucht, die Äußerungen eines ihm völlig fremden Sprechers $\mathrm{zu}$ verstehen. ${ }^{173}$ Obwohl es dabei nur ein Minimum an

171 Vgl. Mayo (1963/64), 144 f. u. Dennett (1971), 102. B. Mayo und Dennett bezeichnen es als ein Dilemma, dass es einerseits nicht möglich ist, frei zwischen wahren und falschen Überzeugungen zu wählen, dass wir andererseits aber auch nicht ausschließlich die Überzeugungen haben, die tatsächlich wahr sind.

172 Vgl. z. B. Davidson (1984), 27, 101, 136 f., 152 f., 168 f., 196 f. u. Davidson (1986), 316. Das Prinzip geht ursprünglich auf N. Wilson zurück. Vgl. Wilson (1958/59), 532. Quine nimmt für sein Gedankenexperiment der ,radikalen Übersetzung“ ein ähnliches Prinzip in Anspruch. Vgl. Quine (1960), 59 f. Ebenso findet sich eine Version bei Dennett. Vgl. Dennett (1971), 93 ff. u. Dennett (1987), 19 ff.

173 Das Gedankenexperiment geht ursprünglich auf Quines „,radikalen Übersetzung“ zurück. Vgl. Quine (1960), Kap. 2. 
Anhaltspunkten gibt, steht dem Interpreten dennoch ein Schlüssel zu Sprache und Denken des fremden Sprechers zur Verfügung. Dieser besteht in der Voraussetzung eines Hintergrundes massiver Übereinstimmung bei den eigenen und fremden Überzeugungen. Und das beginnt damit, dass der Interpret von der Wahrheit der meisten Behauptungen des Fremden ausgeht. Er unterstellt und erwartet, dass der Sprecher größtenteils glaubt, was der Fall ist. Auch aus seiner Sicht sollen die fraglichen Überzeugungen wahr sein.

Dadurch wird also ersichtlich, dass der diskursive Umgang mit Überzeugungen in doppelter Hinsicht normativ aufgeladen ist. Aus der Perspektive der ersten Person entspricht die eigne Überzeugung der Selbstverpflichtung eines Sprechers, dass das, was er behauptet, wahr ist. Er übernimmt gleichsam die Verantwortung für das, was er glaubt und behauptet. Aus der Perspektive der zweiten Person ist eine Überzeugung gleichbedeutend mit einer Erwartung an das Subjekt der Zuschreibung. Wer eine Überzeugung zuschreibt, unterstellt implizit ihre Wahrheit. In beiden Fällen verbindet sich also mit der Überzeugung ein Sollen, das sich an ihren Wahrheitsgehalt richtet.

So gesehen lässt sich Wahrheit als ein normativer Standard begreifen, mit dessen Hilfe über die Angemessenheit einer Überzeugung als solche entschieden wird. ${ }^{174}$ Sie steht dabei weder für eine konkrete Regel in einem präskriptiven Sinne noch für einen bestimmten Regeltypus. Vielmehr ist sie Teil dessen, was das Sprachspiel der Überzeugung in einem allgemeinen, evaluativen Sinne zu einem essentiell normativen macht. An der unmittelbaren Beziehung, die zwischen Wahrheit und Überzeugung besteht, zeigt sich die normative Signifikanz, die den sprachlichen Umgang mit Überzeugungen entscheidend prägt. Denn wie gesehen geht mit jeder behaupteten oder zugeschriebenen Überzeugung stets ein Zugeständnis, eine Verbindlichkeit oder eine Verantwortung einher, welche für diese Form von sprachlicher Performanz konstitutiv ist. Damit tritt eine allgemeine Normativität in den Blick, mit deren Hilfe sich der diskursive Umgang mit Überzeugungen später als Regelfolgen verständlich machen

${ }^{174}$ Vgl. Griffiths (1962/63), 182 ff. Dies ist auch ein zentraler Gedanke in R. Rortys Auseinandersetzung mit dem Wahrheitsbegriff. Vgl. z. B. Rorty (1986), 334 o. Rorty (1988), 16. Vgl. hierzu auch Kap. 6.8 Anm. 319. 
lässt. Wahrheit kann insofern als eine normative Eigenschaft begriffen werden, als sie eine Voraussetzung oder allgemeine Bedingung der Möglichkeit für die Anwendung des Begriffs der Überzeugung ist. ${ }^{175}$

Dieses Wahrheitsverständnis unterscheidet sich jedoch klar von dem, was das Ergebnis tatsächlicher Verifikation ist. Hier geht es um eine Art normative Korrektheit, dort um deskriptive Korrektheit. Vor allem aus der Perspektive der zweiten Person lässt sich dieser Unterschied gut verdeutlichen. Hier kann man klar zwischen physikalischen oder funktionalen Erklärungen auf der einen Seite und intentionalen Erklärungen auf der anderen unterscheiden. ${ }^{176}$ So ermöglicht die Zuschreibung natürlicher Eigenschaften oder Zustände empirische oder deskriptive Aussagen darüber, wie sich jemand oder etwas verhalten wird. Wir stellen beispielsweise fest: „Wenn Du die Glocke bewegst, beginnt sie zu läuten.“ oder „Wer sein Aktiendepot diversifiziert, verringert das Spekulationsrisiko." Die Zuschreibung intentionaler Zustände wie Überzeugungen dagegen erlaubt normative Vorhersagen darüber, wie sich jemand oder etwas verhalten soll. Allerdings ist die Erwartungshaltung, die sich an die Wahrheit einer Überzeugung richtet, zumeist eine implizite. Nur in seltenen Fällen sagen wir Dinge wie: „Wenn ich behaupte, dass mit der Post ein Brief gekommen ist, dann soll das auch so sein.“ oder „Du hast behauptet, dass es Steuererleichterungen geben wird. Also stehe auch dazu!“

Wahrheit als normative Korrektheit zu begreifen, bedeutet somit nicht, dass zwischen einer Überzeugung und irgendwelchen tatsächlichen Gegebenheiten eine notwendige Verbindung bestehen muss. Entscheidend ist nicht, ob eine Überzeugung wahr ist. In pragmatischer Hinsicht vorrangig ist vielmehr, dass sie wahr sein soll. Denn bevor wir beurteilen können, ob eine Überzeugung tatsächlich wahr ist, ist sie bereits mit dem Anspruch, wahr zu sein, geäußert oder zugeschrieben worden. $\mathrm{Zu}$ fragen, ob eine Überzeugung wahr ist, heißt nicht nur $\mathrm{zu}$ fragen, ob sie in deskriptiver Hinsicht in Ordnung ist. Es heißt auch zu fragen, ob sie in normativer Hinsicht in Ordnung ist. ${ }^{177}$ Es geht also nicht nur darum, ob die

\footnotetext{
${ }^{175}$ Vgl. Griffiths (1962/63), 167.

${ }^{176}$ Vgl. Dennett (1971), 97 u. 102 f.

${ }^{177}$ Vgl. Brandom (1994), 17.
} 
Tatsachen dafür sprechen, dass das Geglaubte wahr ist. Es geht auch darum zu beurteilen, ob jemand mit seiner Behauptung der besagten Art von Anspruch gerecht wird, wenn er etwas für wahr hält. Insofern ist eine Wahrheitsbeurteilung immer auch eine normative Beurteilung. Überzeugungen sind immer schon normativ aufgeladen, wenn sie in den Diskurs eingeführt werden.

\subsection{Rationalität normativ verstanden}

Die diskursive Praxis setzt einerseits überwiegende Wahrhaftigkeit voraus. Andererseits verlangt, jemanden als Meinenden bezeichnen und behandeln zu können, ihn als einen überwiegend rationalen Sprecher oder Akteur zu betrachten. Wir schreiben dem anderen Diskursteilnehmer nicht nur zum größten Teil wahre Überzeugungen zu, sondern setzen auch voraus, dass er mit diesen Überzeugungen ein gewisses Maß an logischer Homogenität nicht unterschreitet. Gegebenenfalls sollte er seine Überzeugungen zudem begründen können. Dies sind Erwartungen, die aufzugeben wir nicht leichtfertig bereit sind. Eher noch unterstellen wir eine gestörte Aufmerksamkeit oder Wahrnehmung, oder wir zweifeln an den sprachlichen Kompetenzen, als dass wir die Rationalität einer Person in Frage stellen. Daher ist auch sie Teil des besagten „Nachsichtigkeitsprinzips“.

Was diese Rationalitätsunterstellung im Einzelnen bedeutet, wird sich erst später in vollem Umfang zeigen. Dazu ist es nötig, mehr zu den Eigenheiten logischer Folgebeziehungen zu sagen. ${ }^{178}$ Grob gesagt bedeutet, jemanden als rationalen Sprecher $\mathrm{zu}$ behandeln, dass wir ihm Überzeugungen nach dem allgemeinen Muster gültiger Schlussregeln zuschreiben. Wir schreiben keine widersprüchlichen Überzeugungen zu und unterstellen ein gewisses Maß an deduktiver Übersicht. So schreiben wir jemandem beispielsweise nicht gleichzeitig die Überzeugungen zu, dass der Eiffelturm in Paris steht und dass der Eiffelturm in Pisa steht. Ebenso wenig nehmen wir an, dass jemand glaubt, der Eiffelturm stehe in Paris und Paris sei die Hauptstadt von Frankreich, wenn er sich weigert zu glauben, der Eiffelturm stehe folglich in der Hauptstadt von Frankreich.

${ }^{178}$ Dies werde ich im Rahmen einer inferentiellen Semantik in Kap. 5.7 ff. tun. 
Sicherlich ist in dieser Hinsicht niemand vollkommen rational. Die Rationalitätsannahme ist ein Ideal, das niemand gänzlich erreicht. Wie die Wahrheit ist sie jedoch ein allgemeiner Standard, ohne den es keinen sinnvollen Diskurs geben kann.

Rationalität ist also in erster Linie eine Erwartungshaltung aus der Perspektive der zweiten Person. Genau genommen richtet sie sich aber nicht an die jeweils einzelnen Überzeugungen eines Sprechers, sondern an die Art und Weise, wie sie miteinander in Zusammenhang stehen. Sie ist also immer Merkmal einer Vielzahl von Überzeugungen. Ebenso wenig geht es dabei nur um Überzeugungen. Rationalität betrifft auch denjenigen, der diese hat beziehungsweise dem sie zugeschrieben werden. Als rational bezeichnen wir sowohl ein bestimmtes System von Überzeugungen als auch den Meinenden selbst. Indem wir den inneren Zusammenhang seines Überzeugungssystems beurteilen, beurteilen wir zugleich ihn und können so Vorhersagen treffen, was sein weiteres Denken und Handeln betrifft.

Aus der Perspektive der ersten Person ergibt sich dadurch für denjenigen, der seine Behauptungen äußert, eine weitere Verpflichtung oder Verantwortung. Denn wer etwas behauptet, steht nicht nur für die Wahrheit seiner Überzeugungen ein. Er garantiert auch, dabei die allgemeinen Rationalitätsstandards einzuhalten. So schließt er Widersprüche aus, indem er beispielsweise sagt: „Wenn ich sage ,heute', dann meine ich nicht ,morgen'. “Und er macht Zugeständnisse, was die Gründe und Folgen seiner Behauptung betrifft. Er hält also nicht einfach etwas Einzelnes für wahr, sondern lässt sich auch auf das ein, was daraus folgt und wovon es die Folge ist. Behauptet er beispielsweise, dass es regnet, dann ist er damit zugleich bereit einzuräumen, dass die Straße nass wird. Behauptet er, dass Berlin östlich von Hamburg liegt, so wird er nicht bestreiten, dass Hamburg westlich von Berlin liegt.

Rationalität ist eine grundlegende Erwartung, die wir haben, wenn jemand etwas behauptet oder wenn wir ihm eine Überzeugung zuschreiben. Dies wird auch dann deutlich, wenn wir die Zuschreibung von Überzeugungen mit der von Wünschen kombinieren. ${ }^{179}$ Dabei setzen wir nicht nur die Standards des Schlussfolgerns voraus, sondern überdies, dass

${ }^{179}$ Vgl. auch Dennett (1971), 103. Dennett spricht von dem ,circle of implications between beliefs and desires“. 
der Meinende allgemeinen Grundbedürfnissen wie Überleben, Fortpflanzung, Schmerzfreiheit etc. folgt. Geht man beispielsweise davon aus, dass jemand glaubt, dass der Kochtopf heiß ist und dass er sich nur dann nicht daran verbrennt, wenn er einen Topflappen verwendet, und er zudem wünscht, sich nicht $\mathrm{zu}$ verbrennen, so ergibt sich daraus die Forderung nach der Verwendung eines Topflappens. Überzeugungen und Wünsche bringen also aus der Perspektive des Interpreten die Erwartung eines bestimmten Verhaltens auf Seiten des vermeintlich rationalen Akteurs mit sich. Von der Überzeugung führt eine normative Beziehung zu einer rational erwartbaren Handlung. Wer einen praktischen Schluss nach dem erwähnten Muster zieht, gibt somit Auskunft über das geforderte, nicht aber über das tatsächliche Handeln der betreffenden Person. Die Rationalitätsannahme besagt, dass ein Sprecher oder Akteur sich im Wesentlichen so verhält, wie man es rationalerweise tun sollte. Auch wenn alles für eine bestimmte Handlung spricht, ist jedoch nicht gesagt, dass sie auch letztlich vollzogen wird.

Mit dem Behaupten oder Zuschreiben von Überzeugungen gehen also nicht nur Erwartungen und Verbindlichkeiten einher, die sich an deren Wahrheit richtet. Damit zusammen hängen ebenfalls Einschätzungen, die sich auf die Rationalität der Überzeugungen und den Meinenden selbst beziehen. Rationalität lässt sich daher ebenfalls als ein normativer Standard begreifen, mit dem wir über die Angemessenheit von Überzeugungen und Handlungen entscheiden. Sie gibt vor, welche Überzeugungen ein Sprecher haben und äußern sollte. Dieser Standard ist wesentlich für den sprachlichen Umgang mit Überzeugungen überhaupt sowie für deren spezifische Bedeutsamkeit. Denn aus dieser normativen Signifikanz leitet sich eine normative Form der Korrektheit ab, die sich auf die Folgebeziehungen zwischen verschiedenen Überzeugungen beziehungsweise zwischen Überzeugungen und Handlungen richtet. Daraus ergibt sich für jede Überzeugung ein bestimmter logischer Ort im Netz der Überzeugungen und Handlungen. Und der wiederum fixiert die jeweilige Bedeutung der entsprechenden Aussagen und Ausdrücke. ${ }^{180}$

Die Beispielsätze zur Veranschaulichung der normativen Signifikanz von Überzeugungen mögen bemüht, vielleicht sogar absurd erscheinen. Es

${ }^{180}$ Vgl. hierzu ausführlich Kap. 5. 
ist ungewöhnlich, dass jemand auf die Wahrheit seiner eigenen Behauptung explizit hinweist oder dass sie von einem Zuhörer explizit unterstellt oder eingefordert wird. Eher selten ist auch, dass wir aufgrund bestimmter Überzeugungen explizit darauf schließen, wie sich jemand rationalerweise verhalten sollte. Und dass jemand seine eigenen Überzeugungen und Wünsche auf diese Weise analysiert, klingt geradezu absurd. Zumeist bleibt all dies unausgesprochen. Der springende Punkt ist daher auch, dass die normative Signifikanz im Sprachspiel der Überzeugung zunächst einmal implizit enthalten ist. Was die Beispiele zeigen, ist also nicht viel mehr, als dass die darin thematisierte normative Evaluation vielfach nur um den Preis einer gewissen Manieriertheit explizit gemacht werden kann.

Bei normativen Aus- oder Vorhersagen handelt es sich daher weniger um offene, als vielmehr um nicht artikulierte, innere Erwartungen oder Einschätzungen. Das normative Moment läuft bei Behauptungen und Zuschreibungen von Überzeugungen permanent implizit mit. Insofern ist auch das „Nachsichtigkeitsprinzip“ nichts, worüber wir viel nachdenken und was wir ausdrücklich erwähnen. Allenfalls als rhetorisches Stilmittel kommt es hin und wieder offenen zum Ausdruck. Ansonsten gehen wir stillschweigend davon aus, dass jemand überwiegend wahre Überzeugungen hat und dass er glaubt, was er rationalerweise glauben sollte. Theoretische und praktische Schlüsse hinsichtlich des erwartbaren Denkens und Handelns anderer stellen wir nicht explizit an. Sie sind vielmehr implizit in der diskursiven Praxis enthalten.

Somit wäre es ein Fehler, aufgrund der bisherigen Ausführungen anzunehmen, die Praxis des Behauptens und Zuschreibens von Überzeugungen sei in einem konkreten, präskriptiven Sinne normativ. Nach dem bisher Gesagten sollte man beispielsweise nicht annehmen, dass der Umgang mit Überzeugungen von den folgenden beiden Vorschriften begleitet wird:

$\left(\mathrm{R}_{1}\right)$ Behauptungen und Zuschreibungen von Überzeugungen müssen den Tatsachen entsprechen und somit wahr sein.

$\left(\mathrm{R}_{2}\right)$ Wer Überzeugungen hat, muss rational sein. 
Denn damit wäre der eigentliche Sinn der bisherigen Überlegungen verfehlt. Zum einen würde mit dieser Sichtweise der normative Aspekt des Zusammenhangs von Wahrheit und Überzeugung außer Acht gelassen und ausschließlich in deskriptiver Hinsicht berücksichtigt. Zum anderen würde die Normativität von Überzeugungen insofern falsch verstanden, als versucht würde, sie in Form von expliziten Präskriptionen zu konkretisieren.

Die Idee einer normativen Pragmatik ist nicht die, dass in der diskursiven Praxis Regeln in Form von expliziten, theoretischen Hypothesen befolgt werden. Dieser Vorstellung droht wie gesehen ein infiniter Regress. Die Normativität, die das Behaupten und Zuschreiben von Überzeugungen konstituiert, gilt daher als implizit in der diskursiven Praxis. Die Wahrheits- und Rationalitätsannahme sind lediglich allgemeine Indikatoren von Normativität. Mit ihrer Hilfe lässt sich zeigen, dass es Normativität und normative Korrektheit im Sprachspiel der Überzeugung gibt, nicht aber, wie genau einzelne Regelformulierungen lauten. Der sprachliche Umgang mit Überzeugungen impliziert zunächst nichts weiter als Normativität im Allgemeinen. Das heißt andererseits nicht, dass es dabei keine expliziten Regeln geben kann. Vielmehr ist das Behaupten und Zuschreiben von Überzeugungen bereits normativ signifikant, bevor es zur expliziten Formulierung von Regeln kommt. Wenn daher in den vorausgehenden Abschnitten Begriffe wie Wahrheit, Rationalität, Logik etc. vorkommen, dann nur mit dem methodischen Ziel, die grundlegende Normativität verständlich zu machen. Die Begrifflichkeit nimmt hier einiges vorweg, was die Systematik des anvisierten Theoriemodells eigentlich noch nicht enthält. In den folgenden Abschnitten werde ich diesen Vorgriff insofern rückgängig machen, als ich das Theoriemodell konsequent immanent rekonstruieren werde.

\subsection{Normative Pragmatik}

Im Folgenden werde ich die bisherigen Überlegungen so weiterführen, dass eine übersichtliche Systematik in einer einheitlichen und neutralen Terminologie entsteht. Ziel ist es, die implizit normative Struktur des interpersonalen Austauschs von Überzeugungen sichtbar zu machen. Dabei werde ich vor allem Robert Brandoms normative Rekonstruktion des 
Begriffs der Überzeugung aufgreifen. ${ }^{181}$ Hier ist zunächst noch einmal die Gebrauchsprosa zur Erinnerung: Wer eine Überzeugung hat, hält etwas für wahr. Wer eine Überzeugung zuschreibt, tut in gewisser Weise dasselbe. Er hält für wahr, dass jemand anderes etwas für wahr hält. Was der andere für wahr hält, hält er selbst jedoch nicht zwangsläufig ebenfalls für wahr. Die Zuschreibung bedeutet im Vergleich zur Behauptung einen Perspektivwechsel. Dabei geht es nicht unbedingt um genau dieselbe Überzeugung. Gemein ist den beiden Arten des Umgangs mit Überzeugungen allerdings, dass sie diese zum Gegenstand der Bewertung oder Einschätzung machen. Personen, die etwas behaupten oder zuschreiben, gehen zumeist davon aus, dass es wahr ist: Es soll wahr sein. Die andere Gemeinsamkeit liegt darin, dass, wer eine Überzeugung äußert oder zuschreibt, diese in einen erwartbaren rationalen Zusammenhang stellt. Er stellt Anforderungen was das Verhältnis $\mathrm{zu}$ anderen Überzeugungen angeht: Die Äußerung oder Zuschreibung soll rational sein.

Überzeugungen kommen in der diskursiven Praxis vorrangig auf zwei Art und Weisen vor. Neben dem Behaupten gibt es die Möglichkeit, Überzeugungen zuzuschreiben. Daher ist es möglich und wichtig, den intentionalen Zustand der Überzeugung als den performativen Akt des Behauptens oder Zuschreibens von Überzeugungen $\mathrm{zu}$ betrachten und dabei die normativen Aspekte in den Blick zu nehmen. Mit dem Behaupten oder Zuschreiben kommt einer Überzeugung eine spezifisch normative Eigenschaft zu. Dies ist allerdings keine Eigenschaft, die sie damit erst erwirbt, sondern die sie implizit immer schon hat. Genau genommen ist es auch keine Eigenschaft, die die Überzeugung tatsächlich hat. Vielmehr handelt es sich um etwas, das sie haben soll. Überzeugungen haben die Eigenschaft, auf normative Weise korrekt oder inkorrekt $\mathrm{zu}$ sein beziehungsweise sein zu sollen.

\subsection{Normativer Status}

Die Performanzen des Behauptens oder Zuschreibens von Überzeugungen sind einerseits etwas, was tatsächlich getan wird. Andererseits lassen sie sich auch als etwas beschreiben, was getan werden

${ }^{181}$ Vgl. Brandom (1994), insbes. Kap. 1 u. 2. 
sollte. Sie haben nicht nur deskriptive, sondern auch normative Merkmale. Behauptungen oder Zuschreibungen von Überzeugungen haben eine spezifische normative Kraft. Bezogen auf die Teilnehmer der diskursiven Praxis - diejenigen, die etwas behaupten, zuschreiben oder zugeschrieben bekommen - bedeutet das, dass ihnen mit Blick auf ihre jeweilige Überzeugung etwas zukommt, was man als ,normativen Status“ bezeichnen kann. ${ }^{182}$ Sobald ein Meinender eine Behauptung äußert oder jemand ihm eine Überzeugung zuschreibt, hat er einen solchen Status. Er steht zu einer bestimmten Überzeugung in einer Beziehung, die sich als angemessen oder nicht erweisen kann. Man kann sich dies durch eine Analogie zum Schachspiel verdeutlichen. Bevor der König zum ersten Mal in einer Partie gezogen wird, darf mit ihm - unter bestimmten Bedingungen - eine Rochade ausgeführt werden. Sobald er einmal gezogen wurde, erlischt diese Berechtigung. Man kann also sagen, dass der König mit dem ersten Zug nicht nur seine Position auf dem Brett, sondern auch seinen normativen Status verändert. Er verliert das Recht zu rochieren.

Dies ist der erste Schritt in Richtung praxis-impliziter Normen oder Regeln. Diese sind in der sprachlichen Praxis insofern implizit, als sprachliche Performanzen, in denen Überzeugungen vorkommen, den Diskursteilnehmern normative Status verleihen. Eine Überzeugung zu äußern heißt, einen normativen Status einzunehmen. Eine Überzeugung zuzuschreiben heißt, einen normativen Status zuzuschreiben. Der normative Status eines Diskursteilnehmers korrespondiert mit der normativen Korrektheit oder Inkorrektheit seines sprachlichen Verhaltens. Eine Überzeugung zu äußern heißt, etwas korrekter- oder inkorrekterweise $\mathrm{zu}$ glauben oder meinen. Und eine Überzeugung zuzuschreiben heißt, jemanden als korrekter- oder inkorrekterweise glaubend oder meinend $\mathrm{zu}$ betrachten.

Brandom schlägt vor, den herkömmlichen Begriff der Überzeugung in drei Arten von normativen Status zu unterteilen. ${ }^{183}$ Das sind insbesondere

${ }^{182} \mathrm{Vgl}$. ebd., insbes. 16 f., 33, 41, 57 u. 157 ff. Brandom redet gleichbedeutend vom „normativem“ und „,deontischen Status“. Damit bezeichnet er ein Merkmal nicht nur von Überzeugungen, sondern von jeder Art intentionalem Zustand. Ich werde mich jedoch auf Überzeugungen beschränken.

${ }^{183} \mathrm{Vgl}$. insbes. ebd., $159 \mathrm{f}$. 
die Status der „Festlegung“ und der „Berechtigung“. Die dritte Art ist auf die Beziehung dieser beiden zueinander zurückführbar. Der Status der „Unvereinbarkeit" ergibt sich insofern, als zwei Behauptungen unvereinbar sind, wenn mit der Festlegung auf die eine die Berechtigung zur anderen ausgeschlossen ist. Generell bestehen zwischen Überzeugungen somit sich auseinander ableitende und sich gegenseitig ausschließende Beziehungen. Diese drei Arten von normativen Status entsprechen im Grunde den traditionellen deontischen Modi geboten, erlaubt und verboten. ${ }^{184}$ Allerdings sind sie frei von legalistischen Konnotationen und damit von Zusammenhängen, in denen es um explizit formulierte Regeln und Gesetze geht. Mit einem eigenständigen Vokabular soll der Akzent bewusst auf die implizite Normativität der sprachlichen Praxis gelegt werden. Betrachten wir die normativen Status im Einzelnen.

Eine Festlegung ist ein Status, der jemandem genau dann zukommt, wenn von ihm unter bestimmten Bedingungen eine bestimmte sprachliche Handlung gefordert wird. Das heißt, dass die betreffende Handlung in dem Sinn richtig oder angemessen ist, in dem ihre Unterlassung falsch oder unangemessen wäre. Die Korrektheit der Handlung impliziert die Inkorrektheit ihrer Unterlassung. Man kann auch sagen, dass das, worauf jemand festgelegt ist, für ihn geboten oder verpflichtend ist. Eine bestimmte Überzeugung zu äußern oder zuzuschreiben, legt eine Person darauf fest, weitere Überzeugungen $\mathrm{zu}$ akzeptieren. Wenn jemand beispielsweise behauptet, dass es draußen regnet, dann ist er damit auf die Behauptung festgelegt, dass draußen die Straße nass wird. Schreiben wir jemand die Behauptung zu, dass Berlin östlich von Hamburg liegt, dann ist er damit auf die Behauptung festgelegt, dass Hamburg westlich von Berlin liegt.

Jemand besitzt den Status der Berechtigung genau dann, wenn unter bestimmten Bedingungen eine bestimmte sprachliche Handlung erlaubt ist. Die betreffende, mögliche Handlung ist damit in einem anderen Sinn richtig oder angemessen, als bei der Festlegung. Ihr Vollzug ist korrekt, ohne dass das Ausbleiben gleichzeitig inkorrekt ist. Wir sagen in diesem Fall nicht, die Person sei zu der Handlung verpflichtet, sondern lediglich befugt. Das heißt, dass jemand mit dem Äußern oder Zuschreiben einer

${ }^{184}$ Vgl. Schnädelbach (1992), 85. 
Überzeugung die Befugnis oder den Anspruch erlangt, weitere Überzeugungen $\mathrm{zu}$ äußern oder zuzuschreiben. Behauptet jemand beispielsweise, dass es draußen regnet, dann berechtigt ihn das zu der Behauptung, dass draußen die Temperatur sinken wird. Schreiben wir jemand die Behauptung $\mathrm{zu}$, dass dies ein Glas ist, dann sind wir dazu berechtigt, ihm die Behauptung zuzuschreiben, dass es zerbrechen wird, wenn es herunterfällt. Im Unterschied zur Festlegung ist die Berechtigung jedoch nicht zwingend. Im ersten Beispiel kann es sich auch nur um einen kurzen Regenschauer handeln, der keinerlei Auswirkung auf die Temperatur hat. Und im zweiten Beispiel ist es denkbar, dass das Glas auf einen weichen Untergrund fällt oder derart gehärtet ist, dass es nicht zerbricht.

Der normative Status der Unvereinbarkeit schließlich kommt jemandem genau dann zu, wenn er durch eine bestimmte sprachliche Handlung $\mathrm{zu}$ einer anderen nicht berechtigt ist. Das bedeutet, dass das betreffende Verhalten in dem Sinn richtig oder angemessen ist, in dem ein anderes falsch oder unangemessen wäre. Am deutlichsten wird dies bei gegensätzlichen Überzeugungen. Im diesem Fall ist mit einer Behauptung oder Zuschreibung die Behauptung oder Zuschreibung des Gegenteils verwehrt. Behauptet jemand beispielsweise, dass es draußen regnet, so ist er damit nicht berechtigt zu behaupten, dass draußen die Sonne scheint. Der Status der Unvereinbarkeit bietet darüber hinaus die Möglichkeit, indirekt den Status der Festlegung abzubilden. Man kann nämlich sagen, dass jemand mit einer Behauptung zusätzlich auf genau die Behauptungen festgelegt ist, die mit all denen unvereinbar sind, die auch mit der ursprünglichen Behauptung unvereinbar ist. So ist jemand, der behauptet, dass in Pisa ein um 4,56 Grad in Richtung Südosten geneigter Turm steht, insofern auf die Behauptung festgelegt, dass in Pisa ein schiefer Turm steht, als die beiden Behauptungen mit denselben Behauptungen unvereinbar sind. In beiden Fällen ist beispielsweise ausgeschlossen, dass der Turm „Eiffelturm“ heißt, dass man von ihm aus den Berliner Alexanderplatz sehen kann, dass er zwischen 1973 und 1975 in Toronto errichtet wurde etc. 


\subsection{Normative Einstellung}

Zuschreibungen von Überzeugungen in Ergänzung zu Behauptungen führen einen sinnvollen und notwendigen Perspektivwechsel herbei. Sie erlauben es, die sprachliche Praxis, verstanden als eine soziale Praxis, in ihrer gesamten Ich-Du-Struktur zu berücksichtigen. Die Ich- und die DuPerspektive betreffen nun jedoch in demselben Diskurs nicht unbedingt die gleiche Überzeugung. Genauer gesagt betreffen sie, wenn auch dieselbe Überzeugung, diese nicht unbedingt auf dieselbe Art und Weise. Wenn beispielsweise Karl Hannah die Überzeugung zuschreibt, dass Berlin östlich von Hamburg liegt, so ist damit nicht gesagt, dass Karl dies ebenfalls glaubt. Es bedeutet zwar, dass Hannah nicht nur darauf, sondern auch auf weiteres festgelegt ist, und dass sie auch $\mathrm{zu}$ weiteren Behauptungen berechtigt ist. So ist sie beispielsweise ebenfalls darauf festgelegt, dass Hamburg westlich von Berlin liegt. Zugleich ist sie auch zu der Behauptung berechtigt, dass es zwischen beiden Städten eine Zugverbindung gibt. Das alles gilt für Hannah, nicht jedoch zwangsläufig auch für Karl.

Allgemein gesprochen kommt den verschiedenen Teilnehmern desselben Diskurses nicht automatisch derselbe normative Status zu. Das klingt zunächst trivial, wenn man nur auf Behauptungen schaut. Natürlich behaupten verschiedene Diskursteilnehmer die unterschiedlichsten Dinge. Es geht hier aber nicht vorrangig darum, dass mit den Diskursteilnehmern auch die Überzeugungen variieren. Es geht nicht um den Gehalt einer Überzeugung, sondern um den normativen Zustand, in den sich ein Diskursteilnehmer begibt, wenn er sie ins Gespräch bringt. Besonders im Fall der Zuschreibung ist von Belang, dass unterschiedliche Gesprächsteilnehmer eine unterschiedliche Haltung oder Einstellung hinsichtlich der einzelnen Überzeugungen haben. Genauer noch kann man sagen, dass sie eine unterschiedliche „normative Einstellung“ einnehmen. ${ }^{185}$ Dies lässt sich durchaus in Analogie zu Dennetts Strategie der ,intentionalen Einstellung“ verstehen. Denn die Idee der normativen Einstellung dient ebenfalls der Verdeutlichung der Doppelstruktur der diskursiven Praxis aus Behaupten und Zuschreiben von Überzeugungen,

${ }^{185}$ Vgl. insbes. Brandom (1994), 32 ff., 41 u. 626. 
nun jedoch unter dem Vorzeichen einer bewusst normativen Betrachtung. Diese Perspektivität umfasst die Idee, dass den Diskursteilnehmern unterschiedliche normative Status zukommen, denen wiederum die Einnahme unterschiedlicher normativer Einstellungen entspricht. Hierin liegt eine weitere wichtige Dimension der Normativität im Sprachspiel der Überzeugung.

Zwischen dem normativen Status, der jemandem durch eine Behauptung oder Zuschreibung zukommt, und der normativen Einstellung, die demgegenüber eingenommen wird, besteht ein grundlegender Unterschied. ${ }^{186}$ Der normative Status gibt an, was in normativer Hinsicht richtig oder falsch ist. Das heißt im Einzelnen, er gibt an, worauf jemand festgelegt ist, wozu jemand berechtigt ist und welche seiner Überzeugungen unvereinbar sind. Die normative Einstellung dagegen informiert darüber, was in normativer Hinsicht für richtig oder falsch gehalten wird. Sie gibt an, als worauf festgelegt und als wozu berechtigt oder nicht jemand betrachtet wird. Wenn also beispielsweise Hannah behauptet, dass Berlin östlich von Hamburg liegt, dann betrachtet Karl sie als eben darauf festgelegt und zugleich als zu der Behauptung berechtigt, dass Hamburg westlich von Berlin liegt. Normative Status kommen in der diskursiven Praxis nicht anders vor als durch die jeweiligen Einstellungen der Sprecher und Zuhörer. Etwas salopp formuliert ist das Einzige, was man mit einem normativen Status tun kann, eine normative Einstellung ihm gegenüber einzunehmen. Normative Status werden durch die normativen Einstellungen der Diskursteilnehmer in der Praxis des Diskurses instituiert, oder, noch einmal anders gesagt, sie supervenieren auf normativen Einstellungen. ${ }^{187}$

Der Unterschied von normativen Status und Einstellungen geht also darauf zurück, dass das Sprachspiel der Überzeugung immer eine Angelegenheit unterschiedlicher Perspektiven ist. Die Normativität von Überzeugungen ist das Resultat unterschiedlicher subjektiver Einstellungen. Schließlich gehören Normen oder Regeln nicht zum Bestand der natürlichen Welt der Tatsachen. Sie sind das Werk von uns

\footnotetext{
${ }^{186}$ Vgl. ebd., 41 u. 637.

${ }^{187} \mathrm{Vgl}$. Esfeld (2002), $168 \mathrm{f}$.
} 
Menschen und liegen somit maßgeblich ,im Auge des Betrachters“. ${ }^{188}$ Daher entspricht das Zusammenspiel von normativen Status und Einstellungen der Einsicht, dass Überzeugungen ein soziales Phänomen sind. Normative Status kommen Sprechern und Zuhörern nur durch ihre Teilnahme an der interpersonalen sprachlichen Praxis zu. Normativität gibt es also nur innerhalb einer Gemeinschaft und aus der Perspektive ihrer Mitglieder. Sie kann nicht von außerhalb gegeben sein.

Im Fall des Behauptens ist die Normativität von Überzeugungen vielleicht nicht sofort ersichtlich. Wer etwas behauptet, legt sich auf eine Überzeugung fest. Damit stehen weitere Festlegungen, Berechtigungen und Unvereinbarkeiten in unmittelbarem Zusammenhang. Wieso, so könnte man fragen, sollte dies eine normative Angelegenheit sein? Die diskursive Welt besteht aber nicht nur aus Sprechern, die Behauptungen machen. Es gibt darin ebenso Zuhörer, die Überzeugungen zuschreiben und Behauptungen beurteilen. Man kann dies auch als den Unterschied zwischen dem Einnehmen und dem Zuweisen eines normativen Status bezeichnen. Dann wird ersichtlich, dass sich an Überzeugungen stets normative Erwartungen knüpfen. Beim Einnehmen eines normativen Status entsprechen sich Behauptung und Festlegung. Die Behauptung kann mit dem normativen Status der Festlegung gleichgesetzt werden. Jemandem einen normativen Status zuweisen bedeutet dagegen, ihn als festgelegt, als berechtigt oder als nicht berechtigt zu betrachten. Die Zuweisung und der Status der Festlegung entsprechen sich also nicht zwangsläufig. Das schafft einen Spielraum, der es ermöglicht, zu einer bestimmten Festlegung auf Distanz zu gehen.

Schauen wir uns den zentralen Fall der Festlegung noch etwas genauer an. ${ }^{189}$ Ein Diskursteilnehmer hat hier zwei Möglichkeiten. Auf der einen Seite kann er selbst eine Festlegung eingehen. Er nimmt den normativen Status der Festlegung selbst ein, indem er beispielsweise sagt: „Berlin liegt östlich von Hamburg." Auf der anderen Seite kann der Diskursteilnehmer eine Festlegung jemand anderem zuerkennen. In diesem Fall weist er jemand anderem den Status der Festlegung zu, ohne ihn notwendigerweise selbst anzuerkennen. Er sagt beispielsweise: „Hannah behauptet, dass

\footnotetext{
${ }^{188}$ Vgl. Brandom (1994), 25, 51 f., 161 u. 626.

${ }^{189} \mathrm{Vgl}$. hierzu ebd., $161 \mathrm{ff}$.
} 
Berlin östlich von Hamburg liegt.“ Nur im ersten Fall kommt es also zu einer unmittelbaren Übereinstimmung von Festlegung und besagter Überzeugung beziehungsweise deren Behauptung.

Man kann sich diese beiden Aspekte nun folgendermaßen miteinander verknüpft vorstellen. Wenn jemand eine Festlegung eingeht oder anerkennt, dann berechtigt dies jemand anderen dazu, ihm diese Festlegung zuzuerkennen. Dies ist zunächst unabhängig davon, wie die Dinge wirklich liegen und wie derjenige, der die Festlegung zuerkennt, selbst dazu steht. Wenn er diese Festlegung zugleich jemand anderem zuund für sich selbst anerkennt, kommt dies dem eigenen Eingehen dieser Festlegung gleich. Der andere übernimmt die Festlegung des einen für sich selbst, indem er beispielsweise sagt: „Ich stimme dir zu, dass Berlin östlich von Hamburg liegt." Nun sind andere Diskursteilnehmer dazu berechtigt, ihm diese Festlegung zuzuerkennen etc. Die jeweilige normative Einstellung stellt also für einen Diskursteilnehmer die Möglichkeit dar zu unterscheiden, worauf er sich selbst festlegt und worauf er andere als festgelegt betrachtet. Dadurch ergibt sich ein überaus komplexes und dynamisches Bild, welches die Normativität im Sprachspiel der Überzeugung wiedergibt. Sicher ist dies nur eine unvollständige Skizze. Für das allgemeine Verständnis der normativen Pragmatik ist sie aber ausreichend.

Es lässt sich also festhalten, dass die Normativität von Überzeugen auf zwei allgemeine Momente der diskursiven Praxis zurückgeht: den normativen Status und die normative Einstellung der beteiligten Sprecher. Der sprachliche Umgang mit Überzeugungen versetzt Diskursteilnehmer also unvermeidlich in einen normativen Zustand. Durch Behauptungen und Zuschreibungen von Überzeugungen kommt ihnen der normative Status der Festlegung, der Berechtigung oder der Unvereinbarkeit zu. Verantwortlich für das Vorkommen dieser Status sind die Diskursteilnehmer selbst. Einzig und allein durch normative Einstellungen kommt es dazu, dass normative Status eingenommen oder verliehen werden. 


\subsection{Sanktionen}

Sprecher beurteilen das Behaupten oder Zuschreiben von Überzeugungen anderer Sprecher im Lichte einer Vielzahl von Regeln. Ein explizites Verständnis von Regeln führt jedoch in einen Regress, während Regelmäßigkeiten die Unterscheidung von korrekt und inkorrekt nivellieren. Die Lösung ist, Regeln als implizit in der Praxis zu verstehen. Demnach weisen Sprecher und Zuhörer Verhaltensweisen auf, die so aufgefasst werden können, dass sie das Verhalten anderer implizit als korrekt oder inkorrekt klassifizieren. Anhand des Begriffs der Sanktion lässt sich verdeutlichen, inwiefern das Aufstellen und Erfüllen von Korrektheitsbedingungen als etwas Praxisimplizites verstanden werden kann.

Sanktionen sind einerseits selbst Teil der Praxis, andererseits dienen sie der Institution von Regeln. Dies lässt sich mit Hilfe des Modells des Zuweisens normativer Status systematisch rekonstruieren. Sanktionen sind diesem Modell gewissermaßen inhärent. Denn das gegenseitige Sanktionieren der Diskursteilnehmer lässt sich derart beschreiben, dass sie einander Festlegungen, Berechtigungen und Unvereinbarkeiten zuweisen. Genauer gesagt auferlegen sie sich Festlegungen, erteilen sich Berechtigungen und weisen sich Unvereinbarkeiten nach. Vereinfacht ausgedrückt wendet ein Sprecher eine Sanktion an, indem er dem Adressaten dieser Sanktion ein Recht verleiht oder einer Pflicht auferlegt. Eine Sanktion anwenden ist also nichts anderes, als den normativen Status eines anderen durch die eigene normative Einstellung positiv oder negativ zu beeinflussen oder zu verändern.

Äsops Fabel vom scherzenden Hirten kann dies veranschaulichen:

Ein Hirt, der seine Herde in einiger Entfernung vom Dorf weidete, spielte folgendes Spiel: Er rief nämlich die Dorfbewohner zu Hilfe, weil sich angeblich Wölfe den Schafen näherten. Zwei- oder dreimal erschreckten sich die Dörfler furchtbar, sprangen auf und kehrten mit Gelächter wieder um. Schließlich geschah es, dass wirklich die Wölfe kamen. Seine Herde war abgeschnitten, und der Hirt rief um Hilfe, aber die Dorfbewohner meinten, dass er wie gewöhnlich scherze, und kümmerten sich nicht darum. Und so geschah es, dass er seine Schafe verlor. ${ }^{190}$

190 Äsop (2005), 201. 
Die Fabel zeigt, dass die Dorfbewohner dem Hirten anfangs durchaus glauben. Sie betrachten ihn als darauf festgelegt, dass sich seiner Herde Wölfe nähern. Außerdem gestehen sie ihm weitere Behauptungen zu. Sie betrachten ihn beispielsweise als dazu berechtigt zu behaupten, dass die Wölfe seine Schafe reißen werden, dass die Herde in Gefahr ist, dass die Dorfbewohner die Schafe gemeinsam retten können etc. Indem sie aufspringen und herbeieilen, bestätigen die Dorfbewohner den Hirten in seiner Behauptung. Möglicherweise rufen sie ihm auch explizit zu: „Wenn du behauptest, dass sich deiner Herde Wölfe nähern, dann ist davon auszugehen, dass sie die Schafe reißen werden." Schon das Aufspringen und Herbeieilen lässt sich aber so verstehen, dass sie seine Behauptung positiv sanktionieren.

Schon bald stellt sich aber heraus, dass es verkehrt war, dem Hirten diese Autorität zuzuweisen. Die Dorfbewohner stellen fest, dass er zu der Behauptung „Die Wölfe nähern sich meiner Herde.“ überhaupt nicht berechtigt ist. Folglich betrachten sie ihn auch nicht mehr als dazu berechtigt zu behaupten, dass die Wölfe die Schafe reißen werden, dass die Herde in Gefahr ist, dass die Dorfbewohner die Schafe gemeinsam retten können etc. Die Dorfbewohner halten die Behauptung des Hirten für inkorrekt. Daher ignorieren sie seinen Ruf in Zukunft. Vielleicht kommentieren sie ihn auch mit der Feststellung: „Deiner Herde nähern sich keine Wölfe. Also ist sie auch nicht in Gefahr, und wir müssen sie auch nicht gemeinsam retten." Jedenfalls erfährt das sprachliche Verhalten des Hirten eine negative Sanktionierung. Die Dorfbewohner ändern ihre normative Einstellung ihm gegenüber und weisen ihm einen anderen normativen Status zu.

Die Situation ließe sich noch detaillierter darstellen. Schließlich kommt es nicht nur bei den Dorfbewohnern, sondern auch bei dem Hirten $\mathrm{zu}$ einer Anpassung der normativen Status und Einstellungen. Diese betrachten ihn nicht mehr als zu der Behauptung berechtigt, dass sich seiner Herde Wölfe nähern. Daher betrachtet er sie nicht mehr als darauf festgelegt, dass er zu dieser Behauptung berechtigt ist. Und so wie ihm damit zusammenhängende Berechtigungen entzogen werden, so auch ihnen. Hinzu kommen weitere Festlegungen, Berechtigungen und Unvereinbarkeiten, die den individuellen Hintergrund an Festlegungen und Berechtigungen sowohl der Dorfbewohner als auch des Hirten betreffen. 
Aber auch ohne weitere Details wird deutlich, dass und inwiefern das Behaupten und Zuschreiben von Überzeugungen einer permanenten, wechselseitigen Beurteilung unterliegt, was zu positivem und negativem Sanktionsverhalten führt. Dadurch signalisieren die Diskursteilnehmer, wann eine Behauptung oder Zuschreibung korrekt oder angemessen ist und wann nicht. Und dadurch werden Regeln erkennbar, die in der diskursiven Praxis implizit sind.

Sanktionen stellen eine Unterscheidung zwischen korrekten und inkorrekten diskursiven Praktiken zur Verfügung. Auch hier ist die soziale Dimension von Sprache generell und des Sprachspiels der Überzeugung insbesondere von entscheidender Bedeutung. Schließlich kann sich ein einzelner Sprecher schon allein deswegen nicht korrekt oder inkorrekt verhalten, weil er sein sprachliches Verhalten nicht selbst sanktionieren kann. Er kann versuchen, diese soziale Dimension zu simulieren, etwa indem er ein Selbstgespräch führt. Entscheidend ist aber, was andere für korrekt oder inkorrekt halten und durch ihr Sanktionsverhalten zum Ausdruck bringen. Es braucht also mindestens zwei Diskursteilnehmer, damit das Sprechhandeln zum Gegenstand von sanktionierendem Verhalten werden kann. Was der Eine für korrekt oder inkorrekt hält, steht möglicherweise im Gegensatz zu dem, was in den Augen eines Anderen korrekt oder inkorrekt ist. Worauf dieser sich festlegt, kann etwas sein, als wozu jener ihn nicht berechtigt betrachtet. Einen Standpunkt außerhalb dieses sozialen Perspektivismus jedenfalls gibt es nicht.

Die Praxis der Festlegungs- und Berechtigungszuschreibung ist eine Praxis des Für-korrekt- oder Für-inkorrekt-haltens und manifestiert sich in der sozialen Praxis des wechselseitigen Sanktionierens. Das heißt, weder normative Einstellungen noch normative Status sind von Natur aus manifeste Eigenschaften. Sie sind Teil der Praxis und manifestieren sich indirekt über den Umweg des Sanktionierens. Sanktionen wiederum lösen einen Prozess der wechselseitigen Anpassung aus, dessen Ziel eine Übereinstimmung im Verhalten der beteiligten Personen ist. ${ }^{191}$ Ich werde diesen Prozess der sanktionierenden Anpassung sogleich noch näher erläutern. Grundsätzlich kann man aber bereits festhalten, dass in dieser Herstellung von Übereinstimmung der Ursprung von impliziten Regeln

${ }^{191}$ Vgl. Haugeland (1998a), 147 ff. u. Haugeland (1998b), 310 ff. 
liegt. An der Art und Weise, wie eine bestimmte Reihe von Handlungen in Übereinstimmung mit vorherigen Handlungen gebracht wird, lässt sich ablesen, dass dabei Regeln gefolgt wurde. Die Praxis des Sanktionierens bringt dies zum Vorschein.

\subsection{Interne und externe Sanktionen}

Die Erklärung praxisimpliziter Regeln mit Hilfe von Sanktionen könnte bei kritischen Lesern auf Bedenken stoßen. Diese könnten dadurch begründet werden, dass die Erklärung eine bedrohliche Regressanfälligkeit aufweist. Man könnte ein Problem darin sehen, dass das sanktionierende und das sanktionierte Verhalten von ein und demselben Typ sind. Was etwa der Hirte tut, wenn er behauptet: „Meiner Herde nähern sich Wölfe.“, und was die Dorfbewohner tun, wenn sie erwidern: „Deiner Herde nähern sich keine Wölfe. Also ist sie auch nicht in Gefahr.“, ist schließlich prinzipiell dasselbe. In beiden Fällen handelt es sich um normativ signifikantes Sprachverhalten. Die Behauptungen der Dorfbewohner sanktionieren die Behauptung des Hirten. Wenn aber das Sanktionsverhalten vom selben Typ ist wie das sprachliche Verhalten, über dessen Korrektheit es befindet, dann ist es selbst etwas, das korrekt oder inkorrekt sein kann. Jemand, der etwas sanktioniert, kann somit seinerseits für sein Sanktionieren sanktioniert werden. Dadurch, so der Einwand, kommt ein Regress in Gang. ${ }^{192}$

Um diese Bedenken auszuräumen ist es ratsam, den Begriff der Sanktion genauer zu bestimmen. Dazu können zwei Arten von Sanktionen unterschieden werden: interne und externe. ${ }^{193}$ Interne Sanktionen sind selbst Teil desjenigen Systems, in dem sie zur Anwendung kommen. Sie zählen zu derselben Art von normativ signifikantem Verhalten, zu dessen Spezifikation sie dienen. Daher ist auch ihre Spezifikation nur mit diesen Mitteln möglich, nämlich ebenfalls in normativen Begriffen, das heißt in Begriffen der Korrektheit oder Inkorrektheit. Exterme Sanktionen dagegen können außerhalb des Systems stehen, für dessen Spezifikation sie verwendet werden. Sie zählen zu einer Art von Verhalten, das nicht notwendig normativ signifikant ist. Vielmehr lässt es sich auch in nicht-

192 Vgl. z. B. Rosen (1997), 167 f., Rödl (2000), 770 ff. o. Glüer (2002), 170.

${ }^{193}$ Vgl. Brandom (1994), 42 ff. 
normativen, das heißt in naturalistischen Begriffen spezifizieren. Während also interne Sanktionen Performanzen sind, die selbst wieder von Normen abhängen, sind externe Sanktionen auch nicht-normativ intelligibel.

Durch geringfügige Ergänzungen lassen sich im Beispiel mit dem scherzenden Hirten sowohl interne als auch externe Sanktionen veranschaulichen. So könnten die Dorfbewohner auf das Verhalten des Hirten einerseits reagieren, indem sie ihm die Berechtigung $\mathrm{zu}$ seiner Behauptung sowie zu weiteren, damit zusammenhängenden Behauptungen offen absprechen. Man kann sich das so vorstellen, dass sie sagen: „Deiner Herde nähern sich keine Wölfe. Also ist sie auch nicht in Gefahr." Sie beurteilen seine Behauptung, indem sie ihrerseits Behauptungen formulieren. Damit kommen interne Sanktionen zur Anwendung. Andererseits besagt die Fabel wörtlich, dass sich die Dorfbewohner nach einigen Wiederholungen nicht mehr um die Hilferufe des Hirten kümmern. Wenn er künftig behauptet, dass sich seiner Herde Wölfe nähern, reagieren sie nicht mehr, sondern gehen ihren bisherigen Beschäftigungen nach. Vorstellbar ist zudem, dass sie ihm materielle Strafen auferlegen, die Aufsicht über die Herde entziehen oder gar Stockhiebe erteilen. All dies sind Beispiele für externe Sanktionen. Sie sind nicht zwangsläufig Teil der normativ signifikanten diskursiven Praxis und lassen sich auch ohne Rückgriff auf normatives Vokabular bestimmen.

Die Unterscheidung von internen und externen Sanktionen macht deutlich, dass es sowohl eine normativ signifikante als auch eine nichtnormativ signifikante Art und Weise gibt, etwas als korrekt oder inkorrekt zu behandeln. Die Frage ist nun, ob es Praktiken geben kann, die ausschließlich durch interne Sanktionen bestimmt sind. Dies nämlich wäre die Voraussetzung dafür, dass der erwähnte Regress entstehen kann. Brandom betont ausdrücklich, dass die diskursive Praxis hierfür ein Beispiel ist. ${ }^{194}$ Durch den Verzicht auf externe Sanktionen will er einen Reduktionismus impliziter Regeln auf Regelmäßigkeiten vermeiden. Eine solche Gefahr sieht er, wenn die Korrektheit und Inkorrektheit diskursiver Praktiken auf Sanktionen zurückgeführt wird, die rein naturalistisch bestimmt sind. Ein solches Erklärungsmodell würde - um in McDowells Bild zu bleiben - im Strudel der Charybdis Schiffbruch erleiden. Daher ist

${ }^{194}$ Vgl. ebd., 44 ff. u. 163. 
die Praxis des Eingehens und Zuerkennens von Festlegungen für Brandom so zu verstehen, dass sie mit keinerlei nicht-normativ spezifizierbarem Verhalten in Verbindung steht.

Ich halte dies allerdings für keine treffende Beschreibung. Schließlich ist die diskursive Praxis grundsätzlich in außersprachliche Handlungszusammenhänge eingebettet. Behauptungen haben immer auch die Funktion, auf das Handeln anderer Einfluss zu nehmen oder es mit dem eigenen Handeln zu koordinieren. Das Beispiel vom scherzenden Hirten zeigt, wie auch die offenkundige Weigerung der Dorfbewohner, ihr Handeln auf die Behauptung des Hirten zu stützen, den Charakter einer negativen Sanktion haben kann. In dieser externen Sanktion manifestiert sich die implizite normative Einstellung, den Hirten nicht mehr als zu der Behauptung berechtigt zu betrachten. Das Sanktionsverhalten selbst ist in diesem Zusammenhang zwar normativ signifikant. Es lässt sich aber auch in nicht-normativen Begriffen spezifizieren. Sowohl das Nachgehen ihrer bisherigen Beschäftigung als auch die Stockhiebe sind Verhaltensweisen, die sich in rein naturalistischen Begriffen beschreiben lassen. Brandoms Auffassung, wonach die diskursive Praxis von „Normen bis auf den Grund" geprägt ist, ist also nicht nur regressanfällig, sondern entspricht auch nicht den tatsächlichen Gegebenheiten.

Ein Sprecher behandelt die Überzeugungen eines anderen als korrekt oder inkorrekt, indem er sie positiv oder negativ sanktioniert. Je nachdem wie diese Sanktionen bestimmt werden, besteht die Möglichkeit, weitere Sanktionen darauf anzuwenden oder nicht. Handelt es sich um interne Sanktionen, so können diese wiederum zum Gegenstand weiterer Sanktionen werden. Eine Festlegung ist dann insofern korrekt oder inkorrekt, als sich jemand darauf festlegt, sie für zu anderen Festlegungen berechtigend zu halten oder nicht. Würde man die Praxis des Behauptens und Zuschreibens von Überzeugungen so verstehen, dass sie ausschließlich auf diese wiederum normativ signifikante Art und Weise sanktioniert wird, so könnte man das Erklärungsmodell für regressiv halten. Externe Sanktionen sind für das Modell aber ebenso konstitutiv wie interne. Daher sind derartige Bedenken unbegründet. Da interne Sanktionen prinzipiell durch externe ergänzt werden, gelten letztere also gewissermaßen als Regressbremsen. Man kann sich den Zusammenhang von internen und externen Sanktionen durch eine Art genealogisches oder evolutionäres 
Stufenmodell veranschaulichen. Dazu ist es nötig, noch etwas genauer auf externe Sanktionen eingehen.

Sanktionen etablieren implizite Regeln, indem sie Verhalten bestärken oder unterdrücken. Das geschieht auf verschiedenen Ebenen oder Stufen des Verhaltens. Die komplexeste und elaborierteste Ebene ist die der diskursiven Praxis. Insbesondere wenn es um Überzeugungen geht, ist der Sprachgebrauch normativ signifikant und größtenteils von internen Sanktionen geprägt. Die letzten Abschnitte gaben hierfür eine detaillierte Erklärung. Auf der elementarsten Ebene finden sich dagegen vermehrt externe Sanktionen. Hier geht es im Allgemeinen darum, Verhalten zu konditionieren und so in Übereinstimmung mit einem gültigen Standard zu bringen. Stellt man sich beispielsweise vor, dass die Dorfbewohner dem Hirten wegen seines Verhaltens Stockhiebe erteilen, so kann man dies als den Versuch interpretieren, sein Verhalten an einen solchen Standard anzupassen. Worin dieser besteht, ist selbst nicht Teil der Sanktionen, lässt sich aber so beschreiben, dass Hirten über den Aufenthaltsort von Wölfen grundsätzlich wahre Auskünfte zu machen haben. Das normverletzende Verhalten des Hirten wird durch die Stockhiebe unterdrückt. Sanktionsverhalten in diesem rudimentären Sinn von physischer Bestärkung und Unterdrückung etabliert eine Norm, indem es Übereinstimmungen bei verschiedenen Mitgliedern einer Gemeinschaft zu erzeugen sucht. Typischerweise findet dies durch einen Konditionierungsprozess statt, wenn etwa Ältere Jüngere oder Gleichgesinnte sich gegenseitig erziehen, ausbilden oder trainieren. Dabei gilt, dass Übereinstimmung unterstützt und Abweichung unterdrückt wird. Die normative Einstellung der beteiligten Personen ist sinnlich wahrnehmbar. Das Ziel ist, dass sich der Adressat der Sanktionen in derselben Situation in Zukunft mit größerer oder geringerer Wahrscheinlichkeit auf eine bestimmte Weise verhält.

Externes Sanktionieren ist somit mehr als das bloße Feststellen von Unregelmäßigkeiten. Durch Sanktionen überprüfen und beurteilen die Mitglieder einer Gemeinschaft nicht nur das bisherige Verhalten der Anderen. Sie geben ihnen auch zu verstehen, was sie künftig tun müssen, um sich korrekt $\mathrm{zu}$ verhalten. Sie stellen also tatsächlich Regeln auf. Abweichendes Verhalten ist nicht nur untypisch oder irregulär, sondern inkorrekt, abnormal und inakzeptabel. Es ist etwas, das nicht getan werden 
soll. ${ }^{195}$ Der Rückgriff auf externe Sanktionen bedeutet also keineswegs eine Reduktion praxisimpliziter Normativität auf eine naturalistische Regelmäßigkeitstheorie. ${ }^{196}$ Vielmehr kann dadurch erklärt werden, inwiefern die diskursive Praxis implizit normativ ist, ohne dass dabei selbst normatives Vokabular verwendet wird und ohne dass lediglich nach Regelmäßigkeiten im Verhalten gesucht wird. Sicher sind viele Diskurssituationen nicht unmittelbar in Handlungszusammenhänge eingebunden. Ebenso gibt es Fälle, in denen externe Sanktionen nicht in Bezug zur diskursiven Praxis stehen. Ein empirisch zutreffendes Bild der diskursiven Praxis muss aber einen grundsätzlichen Bezug der normativ signifikanten diskursiven Praxis zu nicht-normativ signifikantem Handeln beinhalten. Insofern ist ein Erklärungsmodell, das sowohl interne als auch externe Sanktionen berücksichtigt, keinem Regress ausgesetzt.

Was nun die Stufenabfolge betrifft, so finden sich $\mathrm{zu}$ unterst grundlegende Formen des externen Sanktionierens. Auf dieser Stufe sind Sanktionen rein physischer Natur. Sie bestärken und unterdrücken Verhalten und erzeugen so Übereinstimmungen innerhalb einer sozialen Gruppierung. Das Verhalten ist auf eine elementare Art normativ signifikant, lässt sich aber durchaus nicht-normativ beschreiben. Auf diese Weise entstehen erste soziale Normen oder Verhaltenskodizes. Externe Sanktionen dieser grundlegenden Art bilden somit die erste und ursprünglichste Stufe, auf der Normativität in der sozialen Praxis vorkommt. Personen erhalten Rechte und Pflichten. Das heißt, es etablieren sich normative Status, die einzelnen oder mehreren Mitgliedern einer sozialen Gruppierung zukommen können.

Auf der nächsten Stufe, wenn normative Status einmal etabliert sind, kommt es zu Beurteilungen und Änderungen dieser normativen Status selbst. Dies geschieht aber immer noch auf eine Weise, die auch in nichtnormativer Hinsicht bestimmt werden kann. Das Verhalten ist normativ signifikant und bezieht sich auf normativ signifikantes Verhalten. Es lässt sich aber durchaus nicht-normativ beschreiben. Eine Person beispielsweise, die unerlaubt eine Schranke durchbricht, wird aufgegriffen

${ }^{195}$ Vgl. Haugeland (1998a), 149.

${ }^{196} \mathrm{Zu}$ der Auffassung, dass auch externes Sanktionieren genuin normative Kraft hat, vgl. auch Haugeland (1998b), 355 f. Anm. 9. Zur Einschätzungen, dass dabei lediglich beliebige Regelmäßigkeiten festgestellt werden, vgl. Brandom (1994), 36. 
und ins Gefängnis gesteckt. Das heißt, nachdem ihr der Zutritt zu einem bestimmten Gelände verwehrt wurde, wird ihr zusätzlich die Berechtigung entzogen, sich innerhalb der Gesellschaft frei zu bewegen. Dieser Freiheitsentzug ist jedoch eine Maßnahme, die im Rahmen der betreffenden Praktiken nicht ausschließlich normativ bestimmt ist.

Auf der letzten Stufe schließlich finden sich interne Sanktionen. Sie dienen der Beurteilung bereits etablierter normativer Status als korrekt oder inkorrekt. Das heißt, das entsprechende Verhalten ist normativ signifikant und kann ausschließlich in normativen Begriffen spezifiziert werden. Paradigmatisch hierfür ist die diskursive Praxis des Behauptens. Sie ist immer auch eine praxisimplizite Form des Sanktionierens. Wie gesehen ist das Äußern und Zuschreiben von Überzeugungen stets normativ signifikant und dient zugleich dazu, weitere Überzeugungen als korrekt oder inkorrekt zu beurteilen. Behauptungen sind gleichzusetzen mit Festlegungen. Demnach geht ein Sprecher mit seiner Behauptung eine Verpflichtung ein, die andere wiederum von ihm einfordern können. Zugleich erteilen sich Sprecher aufgrund ihrer Behauptungen gegenseitig Berechtigungen oder schließen diese gerade aus. So gesehen ist die diskursive Praxis tatsächlich immer ein positives und negatives Beeinflussen ihrer selbst. Diese Form des Sanktionierens von Sanktionen erfolgt prinzipiell aber nur dann, wenn sich auf einer elementaren Ebene externes Sanktionsverhalten ausgebildet hat. Das Sanktionieren interner Sanktionen ist also erst möglich, wenn sich elementare Normen oder Regeln durch externe Sanktionen etabliert und manifestiert haben. Erst wenn es Sanktionen gibt, die auch in naturalistischen Begriffen beschreibbar sind, können Sanktionen, die ausschließlich in normativen Begriffen beschreibbar sind, zum Gegenstand von Sanktionen werden.

Das zentrale Anliegen dieses Kapitels war, die Normativität im sprachlichen Umgang mit Überzeugungen aufzuzeigen. Zu diesem Zweck habe ich im ersten Teil zunächst den Begriff der Überzeugung auf zwei pragmatische Grundformen zurückgeführt. Ich habe Behauptungen und Zuschreibungen als die elementarsten Formen des diskursiven Umgangs mit Überzeugungen ausgewiesen. Damit habe ich der allgemeinen Einsicht Rechnung getragen, dass Sprache grundsätzlich eine soziale Praxis ist. Daraufhin ging es darum zu zeigen, dass und inwiefern die Praxis des 
Behauptens und Zuschreibens von Überzeugungen normativ signifikant ist. Ich habe einerseits geltend gemacht, dass damit immer ein Anspruch und ein Erwartung einhergeht, was die Wahrheit der fraglichen Überzeugung angeht. Dies habe ich als eine erste Form der normativen Signifikanz oder Aufgeladenheit von Überzeugungen beschrieben. Andererseits habe ich dargelegt, dass insbesondere mit dem Zuschreiben von Überzeugungen eine Erwartung einhergeht, was deren Rationalität betrifft. Daher habe ich diese allgemeine Rationalitätsunterstellung als eine weitere Form der normativen Signifikanz bezeichnet.

Eine detaillierte, systematische Rekonstruktion der normativen Pragmatik war das Anliegen des zweiten Teils dieses Kapitels. In einer Terminologie, die im Kern auf Brandom zurückgeht, habe ich den sprachlichen Umgang mit Überzeugungen als eine durch und durch normative Angelegenheit dargestellt. Ich habe ihn so charakterisiert, dass Sprechern dabei stets normative Status zukommen. Dies aber nur insofern, als sie anderen Sprechern und dem, was sie behaupten, gegenüber normative Einstellungen einnehmen. Somit ist das Sprachspiel der Überzeugung als ein komplexes Geflecht aus Festlegungen, Berechtigungen und Unvereinbarkeiten zu begreifen. Sprecher haben nicht einfach Überzeugungen, sondern sie bewerten sich gegenseitig permanent, sobald ihre Überzeugungen in den Diskurs eintreten. Dabei habe ich die Tatsache hervorgehoben, dass dies eine Angelegenheit ist, die implizit in der diskursiven Praxis ist. Das habe ich damit plausibilisiert, dass ich das praktische Unterscheiden in korrekt und inkorrekt auf den Begriff der Sanktion zurückgeführt habe. Denn durch sanktionierendes Verhalten entsteht genau die Form von normativer Korrektheit, die auf implizite Regeln schließen lässt.

Das Sprachspiel der Überzeugung ist immer ein normatives Sprachspiel. Behaupten und Zuschreiben von Überzeugungen sind diskursive Praktiken, die stets normativ aufgeladen sind. Der Umgang mit Überzeugungen bringt für die Diskursteilnehmer stets Rechte und Pflichten mit sich. Daher ist es gerechtfertigt zu sagen, dass der Austausch von Überzeugungen an impliziten Regeln orientiert ist. Das hat allerdings Konsequenzen für das, was wir unter propositionalem Wissen verstehen. Denn wenn Überzeugungen ein elementarer Bestandteil von Wissen sind und wenn der Umgang mit Überzeugungen wiederum im Wesentlichen 
regelgeleitet ist, dann ist es auch der Umgang mit Wissen. Das heißt, wenn bereits beim Umgang mit Überzeugungen Regeln befolgt werden, dann liegt dem eine praktische Fähigkeit zugrunde, die auch grundlegender als Wissen ist. Dies ist es, was ich als Können bezeichne. Bereits der Umgang mit Überzeugungen ist eine Form von Können. 
